\title{
Stimulation of lymphangiogenesis via VEGFR-3 inhibits chronic skin inflammation
}

\author{
Reto Huggenberger, ${ }^{1}$ Stefan Ullmann, ${ }^{1}$ Steven T. Proulx, ${ }^{1}$ Bronislaw Pytowski, ${ }^{2}$ \\ Kari Alitalo, ${ }^{3}$ and Michael Detmar ${ }^{1}$ \\ ${ }^{1}$ Institute of Pharmaceutical Sciences, Swiss Federal Institute of Technology, ETH Zurich, 8093 Zurich, Switzerland \\ ${ }^{2}$ Imclone Systems, New York, NY 10014 \\ ${ }^{3}$ Molecular/Cancer Biology Laboratory, Biomedicum Helsinki, University of Helsinki, Fl-00014 Helsinki, Finland
}

\section{CORRESPONDENCE}

Michael Detmar:

michael.detmar@pharma.ethz.ch

Abbreviations used: K14, keratin 14 ; $\mathrm{Tg}$, transgenic; VEGF, vascular endothelial growth factor; VEGFR, VEGF receptor.
Pathological angiogenesis and lymphangiogenesis have received increasing interest, mainly because of their presumed role in enhancing tumor progression and metastasis (Carmeliet, 2003; Hirakawa et al., 2005; Karpanen and Alitalo, 2008; Mumprecht and Detmar, 2009). However, vascular remodeling is also a hallmark of many inflammatory diseases such as chronic airway inflammation, rheumatoid arthritis, inflammatory bowel disease, and the chronic inflammatory skin disease psoriasis (Detmar et al., 1994; Baluk et al., 2005; Bainbridge et al., 2006; Danese et al., 2006). In these conditions, levels of vascular endothelial growth factor (VEGF) A are elevated in the inflamed tissue (Detmar et al., 1994; Koch et al., 1994; Kanazawa et al., 2001). Homozygous keratin 14 (K14) VEGF-A transgenic (Tg) mice, which overexpress mouse VEGF- $\mathrm{A}_{164}$ in the epidermis, spontaneously develop a chronic inflammatory skin disease with many features of human psoriasis at an age of $\sim 6$ mo (Xia et al., 2003).
In hemizygous K14-VEGF-A Tg mice, chronic inflammatory skin lesions can be induced by delayed-type hypersensitivity reactions (Kunstfeld et al., 2004), and we have previously used this model to discover that topical application of a small molecule inhibitor of VEGF receptor (VEGFR) kinases results in potent antiinflammatory effects that were subsequently also found in other models of inflammation (Halin et al., 2008). Specific inhibition of VEGF-A also ameliorated psoriasis-like symptoms in a mouse model of psoriasis, where the epidermal specific deletion of c-Jun and JunB leads to the disease (Schonthaler et al., 2009). Together, these results indicate an important role of angiogenesis and blood vascular activation in sustaining chronic inflammation.

0 2010 Huggenberger et al. This article is distributed under the terms of an Attribution-Noncommercial-Share Alike-No Mirror Sites license for the first six Attribution-Noncommercial-Share Alike-No Mirror Sites license for the first six
months after the publication date (see http://www.rupress.org/terms). After six months it is available under a Creative Commons License (Attribution-Noncommercial-Share Alike 3.0 Unported license, as described at http://creativecommons .org/licenses/by-nc-sa/3.0/). 
A

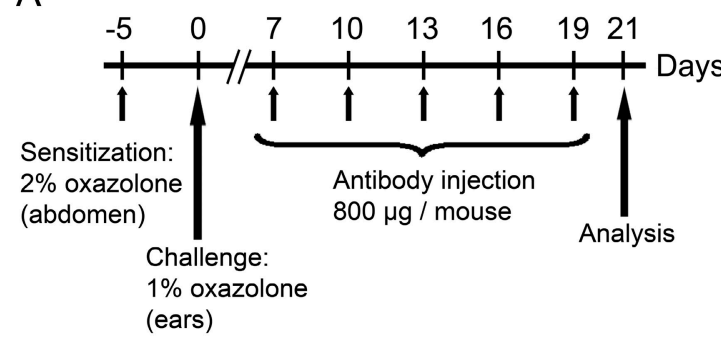

$B \cdot \diamond-\operatorname{IgG} \rightarrow-$ anti-VEGFR-2 $\neg$ anti-VEGFR-3

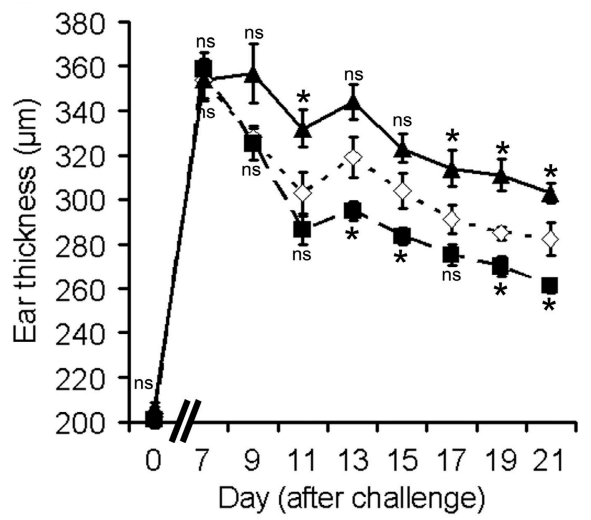

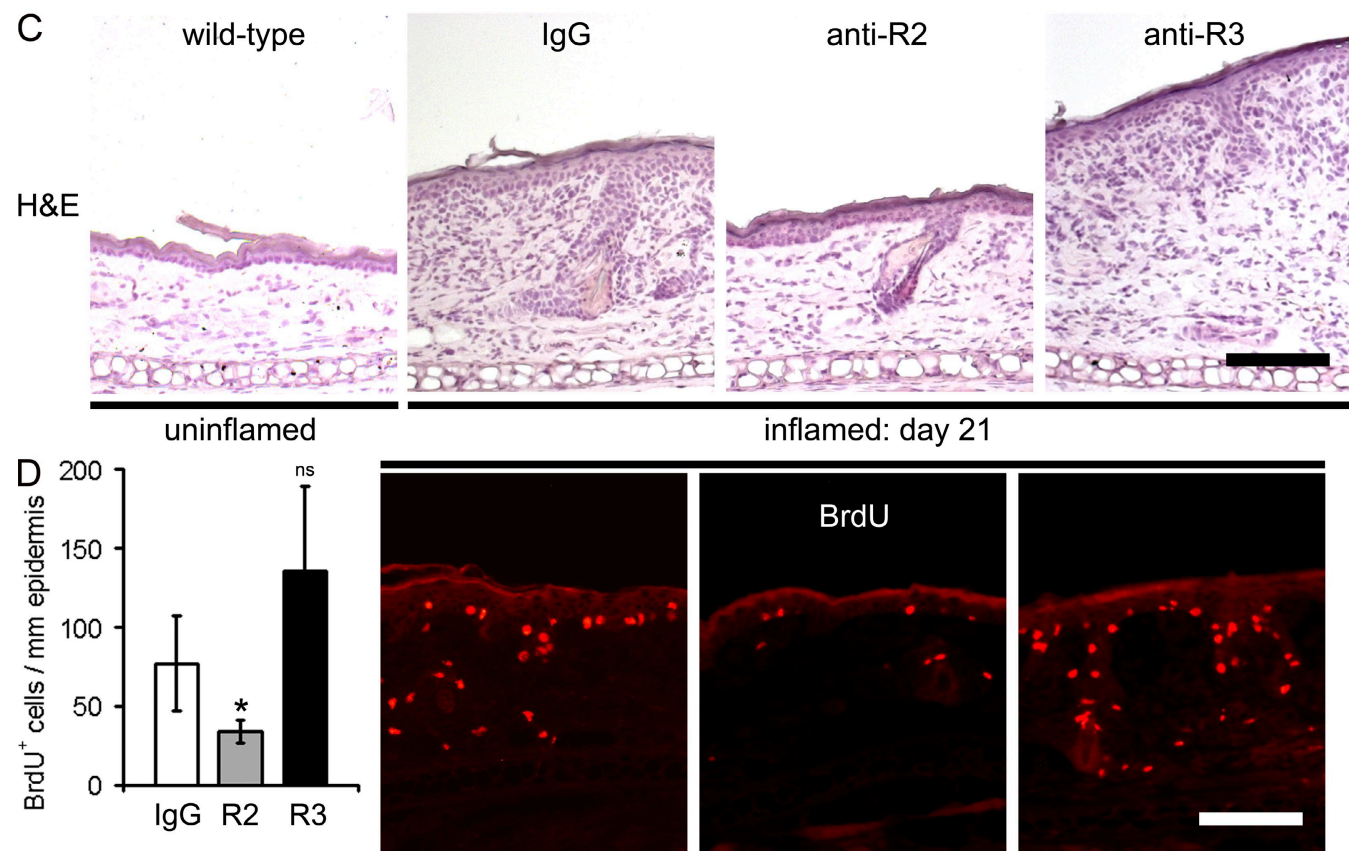

Figure 1. Systemic inhibition of VEGFR-3 prolongs edema formation in chronic skin inflammation. (A) Hemizygous K14-VEGF-A Tg mice ( $n=15)$ were painted at day -5 with $2 \%$ oxazolone and challenged at day 0 with $1 \%$ oxazolone on the ears. Starting at study day 7 , the mice received a total of five i.p. injections of mAb DC101 (anti-mouse VEGFR-2), mF4-31C1 (anti-mouse VEGFR-3), or control rat lgG ( $n=5$ per group) every $3 \mathrm{~d}$. Two independent experiments were performed. (B) Treatment with mF4-31C1 $(\mathbf{\Delta})$ resulted in prolonged ear swelling, as compared with IgG-treated controls $(\diamond)$, whereas DC101 ( $\mathbf{\square})$ treatment reduced the inflammatory ear swelling. Data represent mean \pm SEM. (C) Hematoxylin and eosin (H\&E) stains of mouse ears at day 21 after oxazolone challenge revealed increased edema formation, epidermal hyperthickening, and cell infiltration in the lgG-treated control group and after anti-VEGFR-3 mAb treatment, as compared with untreated wild-type mice $(n=3)$. Anti-VEGFR-2 mAb injection largely normalized skin architecture. One ear-half is shown. (D) Immunofluorescence stainings and quantification of BrdU+ cells show similar numbers of proliferating cells in the ear skin after anti-VEGFR-3 (R3) mAb treatment compared with IgG-injected mice. Systemic inhibition of VEGFR-2 (R2) significantly reduced the number of $\mathrm{BrdU}+$ cells. Data represent mean $\pm \mathrm{SD}$. ${ }^{*}, \mathrm{P}<0.05$. ns, not significant. Bars, $100 \mu \mathrm{m}$.

In contrast, the role of the lymphatic vasculature in chronic inflammation has remained unclear.

It has been reported that the lymphatic vasculature plays an active role in corneal and kidney transplant rejection, in part by facilitating dendritic cell transport to draining lymph nodes (Cursiefen et al., 2004; Kerjaschki et al., 2004). In contrast, specific blockade of VEGFR-3, a receptor for the lymphangiogenic growth factors VEGF-C and VEGF-D which is mainly expressed on the lymphatic endothelium in the adult (Kaipainen et al., 1995), increased edema formation in a mouse model of chronic airway inflammation (Baluk et al., 2005). Moreover, lymphatic vessels have an increased density in arthritic joints of mice and men and are further increased after standard infliximab therapy (Zhang et al., 2007; Polzer et al., 2008). In inflamed tissues, the lymphangiogenic factors VEGF-C and VEGF-A are secreted by immune cells such as macrophages and by resident tissue cells such as keratinocytes and fibroblasts. After proteolytic processing of the propeptides, the mature VEGF-C also binds and activates VEGFR-2 which, besides its expression on the blood vascular endothelium, is also expressed 

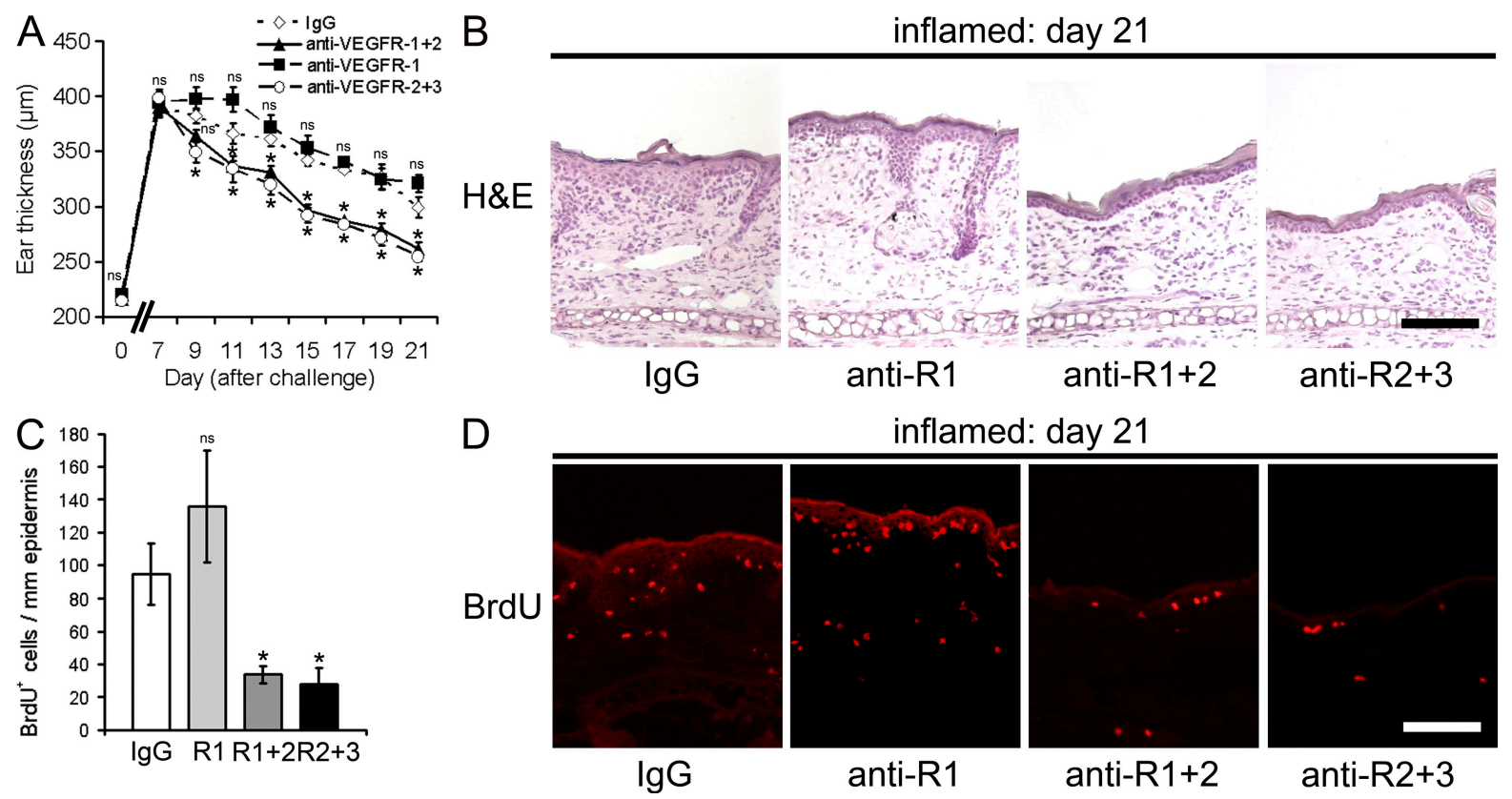

inflamed: day 21

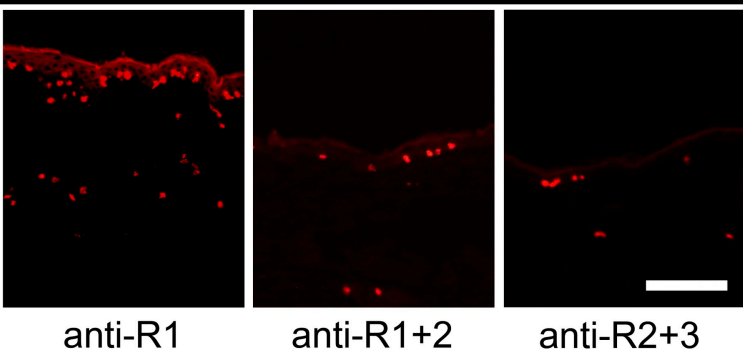

Figure 2. Systemic inhibition of VEGFR-1 does not reduce chronic skin inflammation. (A) Hemizygous K14-VEGF-A Tg mice $(n=20)$ were painted and challenged as described in Fig. 1. Starting at study day 7, mice received five i.p. injections of mAb MF1 (anti-VEGFR-1, $\mathbf{\square}$ ), MF1 + DC101 (anti-VEGFR-1+2, $\mathbf{\Delta}$ ), DC101 + mF4-31C1 (anti-VEGFR-2+3, $)$ ), or control rat $\operatorname{lgG}(\diamond, n=5$ per group) every $3 \mathrm{~d}$. Treatment with MF1 did not affect the chronic inflammatory response, whereas combined treatment with DC101 + MF1 and with DC101 + mF4-31C1 potently reduced skin inflammatory ear swelling. Data represent mean \pm SEM. Two independent experiments were performed. (B) H\&E stains of mouse ears at study day 21 after oxazolone challenge revealed edema formation, epidermal thickening, and inflammatory cell infiltration in the lgG control group and after anti-VEGFR-1 mAb treatment. Anti-VEGFR-1+2 and anti-VEGFR-2+3 treatment largely normalized skin architecture. One ear half is shown. (C and D) Immunofluorescence stainings and quantification of BrdU+ cells show similar numbers of proliferating cells in the ear skin after anti-VEGFR-1 (R1, anti-R1) mAb treatment compared with control IgG-injected mice. Systemic inhibition of VEGFR-1+2 (R1+2, anti-R1+2) or VEGFR-2+3 (R2+3, anti-R2+3) reduced the number of $\mathrm{BrdU}+$ cells. Data represent mean $\pm \mathrm{SD} .{ }^{*}, \mathrm{P}<0.05$. ns, not significant. Bars, $100 \mu \mathrm{m}$.

on lymphatic vessels (Joukov et al., 1997; Kriehuber et al., 2001; Mäkinen et al., 2001b; Wirzenius et al., 2007).

We have recently found that lymphatic vessels are enlarged in human psoriasis skin lesions and that lymphangiogenesis is also a characteristic feature of the K14-VEGF-A chronic skin inflammation Tg mouse model (Kunstfeld et al., 2004). Importantly, the K14-VEGF-A Tg mice are sensitive to standard antipsoriatic therapies, such as betamethasone, and they develop a Th17-like disease phenotype that is similar to human psoriasis (Hvid et al., 2008). In the present study, we used this model to investigate the individual contribution of the three VEGFRs to angiogenesis, lymphangiogenesis, and inflammation and the role of lymphatic vessels in chronic skin inflammation.

To this end, we first treated K14-VEGF-A Tg mice during the chronic phase of induced skin inflammation with blocking antibodies against VEGFR-1, -2 , or -3 , individually or in combination. In a second genetic approach, we established double $\mathrm{Tg}$ mice with overexpression of both VEGF-A and VEGF-C in the epidermis (K14-VEGF-A+C Tg mice), and also K14-VEGF-A/VEGF-D double Tg mice, and compared the course of induced skin inflammation in these mice with that observed in K14-VEGF-A single Tg mice. Overall, our studies reveal that VEGFR-2 is the main mediator of VEGF-Ainduced pathological angiogenesis, lymphangiogenesis, and chronic skin inflammation. Unexpectedly, we also identified an important role of VEGF-C-induced lymphatic vessel activation in reducing the characteristic signs of cutaneous inflammation and in preventing the development of chronic inflammation. Importantly, the antiinflammatory effect of VEGF-C was seen both in a genetic mouse model with chronic overexpression of VEGF-C in the skin and after intracutaneous injection of recombinant VEGF-C protein. These studies, together with results in K14/VEGF-A/-D double Tg mice, also revealed that the antiinflammatory effects are mediated via activation of VEGFR-3. We hypothesize that stimulation of functional lymphangiogenesis - in addition to anti-angiogenic therapies - might represent a novel strategy to treat chronic inflammatory disorders of the skin or possibly also other chronic inflammatory diseases.

\section{RESULTS}

\section{Systemic blockade of VEGFR-3 prolongs inflammatory} edema formation

We first investigated the distinct roles of VEGFR-2 versus VEGFR-3 in chronic inflammation, using hemizygous K14VEGF-A Tg mice that develop chronic skin inflammation after sensitization and challenge with the contact sensitizer oxazolone (Kunstfeld et al., 2004). K14-VEGF-A Tg mice were painted with oxazolone (study day -5 ) and, $5 \mathrm{~d}$ later, 


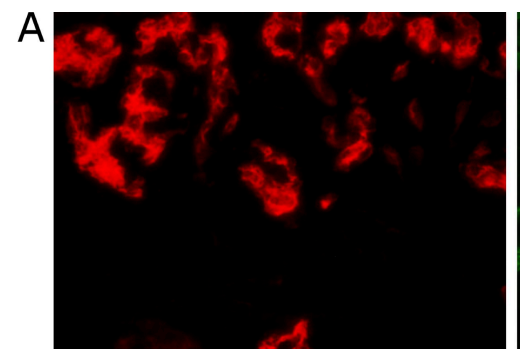

MECA-32

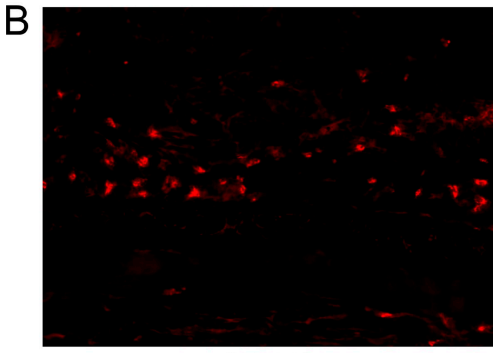

CD11b

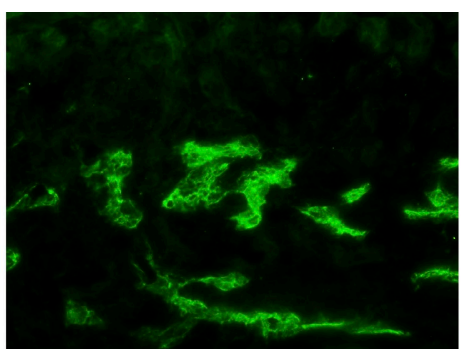

VEGFR-3

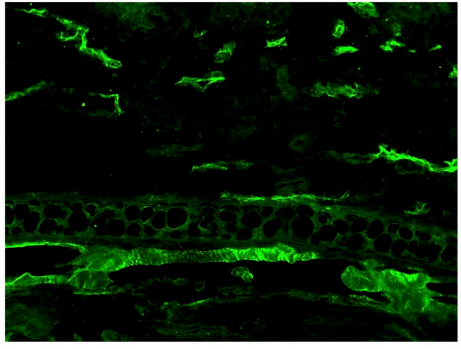

VEGFR-3

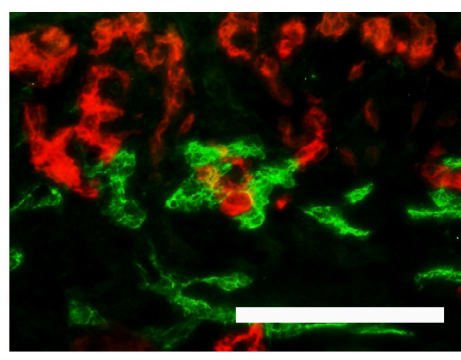

Merge

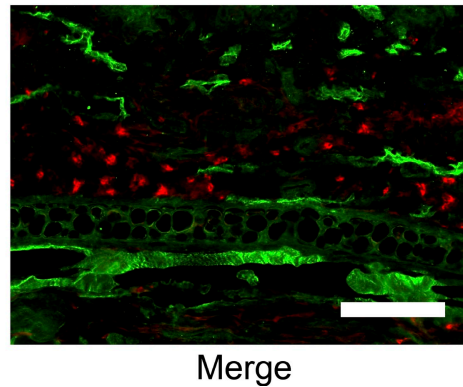

Figure 3. VEGFR-3 expression is restricted to lymphatic vessels in the inflamed skin of K14-VEGF-A Tg mice. (A) Differential immunofluorescence analyses for the blood vessel-specific marker MECA-32 (red) and for VEGFR-3 (green) revealed strong expression of VEGFR-3 by MECA-32-negative lymphatic vessels, whereas VEGFR-3 expression was largely absent on MECA-32-positive blood vessels. (B) CD11 b+ cells (red) lack expression of VEGFR-3 (green). Ear skin of IgG-injected K14-VEGF-A Tg mice at $21 \mathrm{~d}$ after oxazolone challenge is shown. The pictures shown are representative of five mice. Two independent experiments were performed. Bars, $100 \mu \mathrm{m}$.

were challenged on both sides of the ears by topical application of oxazolone (Fig. 1 A). Starting at day 7 after challenge, the mice received five i.p. injections of either the inhibitory anti-VEGFR-2 mAb DC101 or the anti-VEGFR-3 mAb mF4-31C1 every $3 \mathrm{~d}$, whereas the control group received isotype-matched IgG. All mice were analyzed $2 \mathrm{~d}$ after the last antibody injection at study day 21 (Fig. 1 A).

Systemic inhibition of VEGFR-2 significantly reduced inflammatory ear swelling, as compared with control IgGinjected mice (Fig. 1 B). Surprisingly, blockade of VEGFR-3 significantly prolonged the inflammatory ear swelling (Fig. 1 B). Ear tissue sections of control IgG injected mice showed the typical epidermal hyperplasia (acanthosis) and inflammatory cell infiltration at $21 \mathrm{~d}$ after oxazolone challenge, as compared with uninflamed wild-type mice (Fig. 1 C). The systemic inhibition of VEGFR-2, but not of VEGFR-3, diminished edema formation, epidermal hyperplasia, and inflammatory cell infiltration in the skin (Fig. 1 C). Inhibition of VEGFR-2 also reduced the number of proliferating cells by $44 \%$, as compared with control IgG-treated mice $(\mathrm{P}<0.05$; Fig. $1 \mathrm{D})$, whereas no significant effect was seen after systemic blockade of VEGFR-3.

\section{Systemic blockade of VEGFR-1 does not inhibit chronic skin inflammation}

We next painted and challenged K14-VEGF-A Tg mice with oxazolone and inhibited either VEGFR-1 alone or VEGFR-1+2 or VEGFR-2+3 in combination (Fig. 1 A). Systemic blockade of VEGFR-1 did not significantly change inflammatory ear swelling, whereas the combined inhibition of VEGFR-1+2 and VEGFR-2+3 strongly reduced ear thickness (Fig. 2 A). At the histological level, skin sections of IgG-injected and anti-VEGFR-1 mAb-treated mice showed a comparable epidermal hyperplasia and dermal inflammatory cell infiltration at $21 \mathrm{~d}$ after oxazolone challenge. In contrast, anti-VEGFR-1+2and-VEGFR-2+3-injected mice showed reduced thickening of the epidermis and decreased inflammatory cell infiltration (Fig. 2 B). After systemic blockade of VEGFR-1, there was no difference in the number of proliferating $\mathrm{BrdU}^{+}$cells as compared with IgG injection (Fig. 2, C and D). In contrast, inhibition of VEGFR-1+2 or VEGFR-2+3 strongly reduced the number of proliferating cells by 65 or $71 \%$, respectively, as compared with IgG-treated mice (both $\mathrm{P}<0.001$; Fig. 2, C and D).

\section{VEGFR-2 is the main mediator of VEGF-A-induced inflammatory angiogenesis}

At $21 \mathrm{~d}$ after oxazolone challenge, the number of MECA-32+ cutaneous blood vessels per millimeter of epidermal basement membrane was increased by $316 \%$ in control IgG-treated K14VEGF-A Tg mice as compared with untreated wild-type mice, and blood vessel size was increased by $230 \%$ (Fig. S1 A). Systemic inhibition of VEGFR-2, VEGFR-1+2, and VEGFR-2+3 significantly reduced blood vessel number and size, whereas inhibition of VEGFR-1 or VEGFR-3 alone did not affect the blood vessel number or the blood vessel size (Fig. S1, A and B).

Because expression of VEGFR-3 has been previously detected on some blood vessels associated with tumors and healing wounds (Valtola et al., 1999; Paavonen et al., 2000), we next 

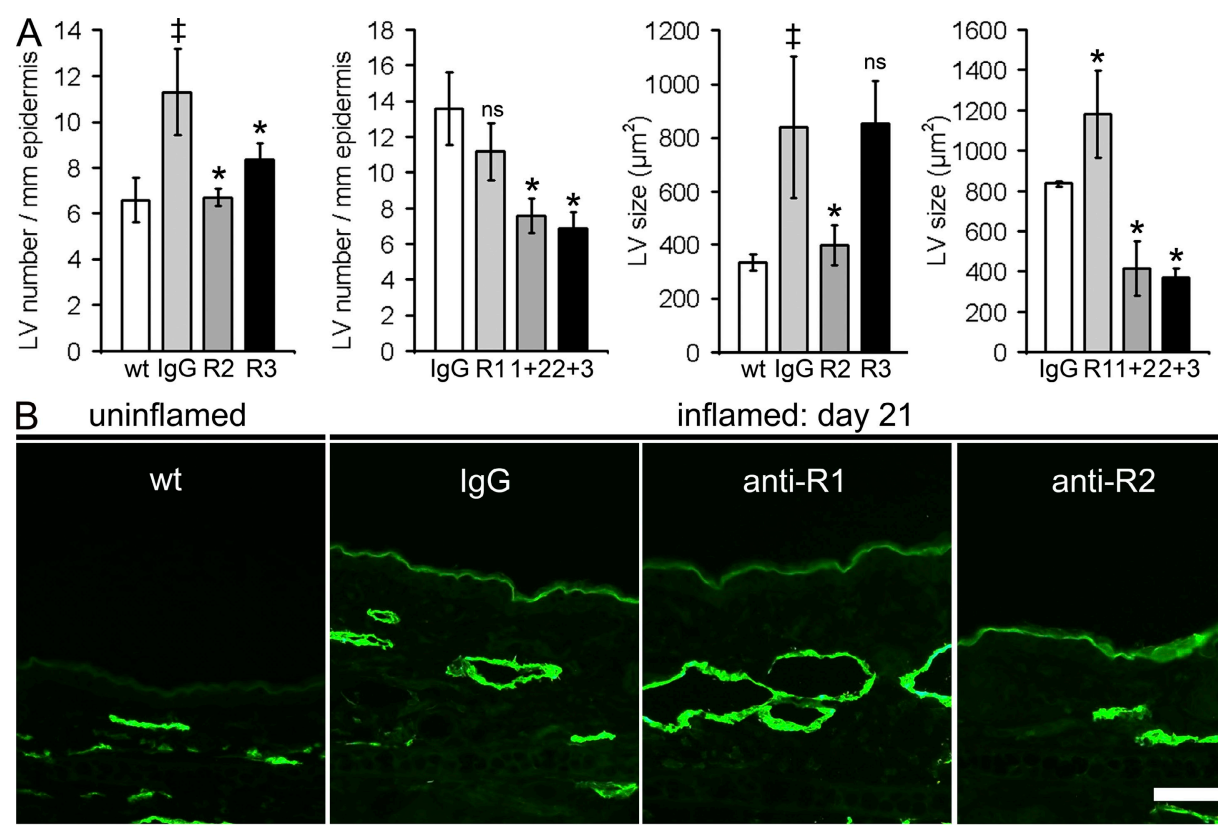

inflamed: day 21
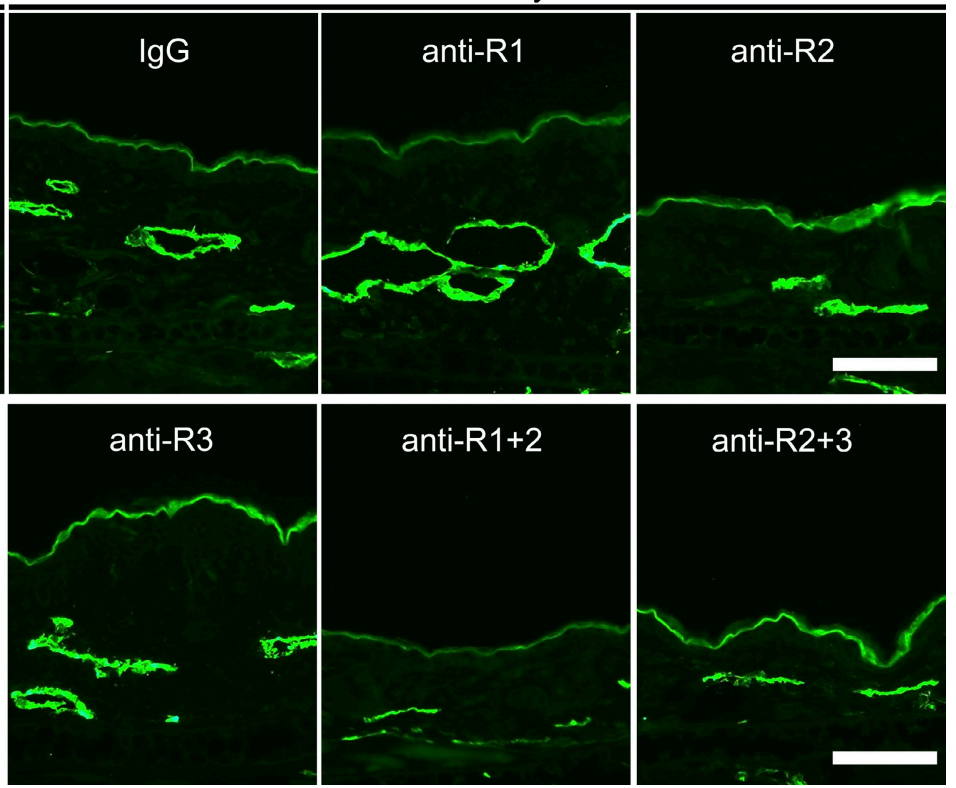

Figure 4. Inflammatory lymphangiogenesis is inhibited by blockade of VEGFR-2 or of VEGFR-3. (A) Quantitative image analyses of lymphatic vessels (LV; LYVE-1+, green) revealed a significantly increased number and size of lymphatic vessels in control lgG injected mice at day 21 after induction of inflammation, as compared with normal wild-type (wt) mice. The mean number of lymphatic vessels was reduced after anti-VEGFR-2 (R2) and after anti-VEGFR-3 (R3) treatment but not after systemic inhibition of VEGFR-1 (R1). Combined inhibition of VEGFR-1+2 (1+2) or VEGFR-2+3 (2+3) also reduced lymphatic vessel numbers. Systemic inhibition of VEGFR-2, VEGFR-1+2, and VEGFR-2+3 resulted in a reduced mean size of lymphatic vessels. $n=5$ mice per group. Two independent experiments were performed. Data represent mean \pm SD. $\neq, P<0.05$ versus wild-type; ${ }^{*}, P<0.05$ versus $\operatorname{lgG}$. ns, not significant. (B) Representative images of LYVE-1 immunostains (green) are shown. Bars, $100 \mu \mathrm{m}$.

assessed VEGFR-3 expression on inflamed blood vessels. In agreement with the lack of blood vessel changes after antiVEGFR-3 treatment, we found that the expression of VEGFR-3 was restricted to lymphatic vessels in the chronically inflamed skin of hemizygous K14-VEGF-A Tg mice (Fig. 3, A and B).

\section{VEGFR-2 and -3 mediate VEGF-A-induced inflammatory lymphangiogenesis}

Computer-assisted morphometric analyses of skin sections, stained for the lymphatic-specific marker LYVE-1 at study day 21, revealed that lymphatic vessel number and lymphatic vessel size were increased by 171 and 251\%, respectively, in the inflamed skin of control IgG-treated K14-VEGF-A Tg mice as compared with untreated wild-type mice (Fig. 4, A and B). Anti-VEGFR-3 $\mathrm{mAb}$ treatment and, more efficiently, anti-VEGFR-2 treatment diminished the number of lymphatic vessels (Fig. 4, A and B). Importantly, we found that VEGFR-2 was expressed on lymphatic vessels in normal skin of wild-type mice and in inflamed skin of K14-VEGF-A Tg mice (Fig. S2). Anti-VEGFR-2 treatment also resulted in a normalization of lymphatic vessel size, whereas the lymphatic vessels remained dilated in the anti-VEGFR-3-treated group (Fig. 4, A and B). The inhibition of VEGFR-1 did not affect the number of lymphatic vessels but slightly increased their caliber (Fig. 4, A and B). Inhibition of VEGFR-1+2 and of VEGFR-2+3 in combination normalized both the lymphatic vessel number and the lymphatic vessel size to the levels observed in normal uninflamed wild-type mice (Fig. 4, A and B).

We next used TaqMan-based real-time RT-PCR to investigate the levels of transcripts for the lymphangiogenic factors VEGF-C and VEGF-D. We found that VEGF-C transcript levels were significantly increased by $38 \pm 20 \%$ in the inflamed skin of IgG-injected mice at $21 \mathrm{~d}$ after oxazolone challenge, as compared with uninflamed wild-type mice $(\mathrm{P}<0.05)$, whereas VEGF-D levels were unchanged. These data indicate that VEGF-C levels are up-regulated in 
A uninflamed inflamed: day 21
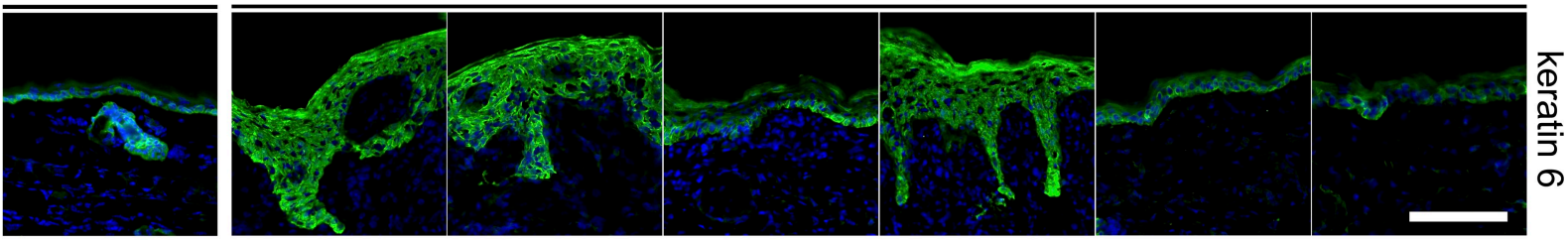

B
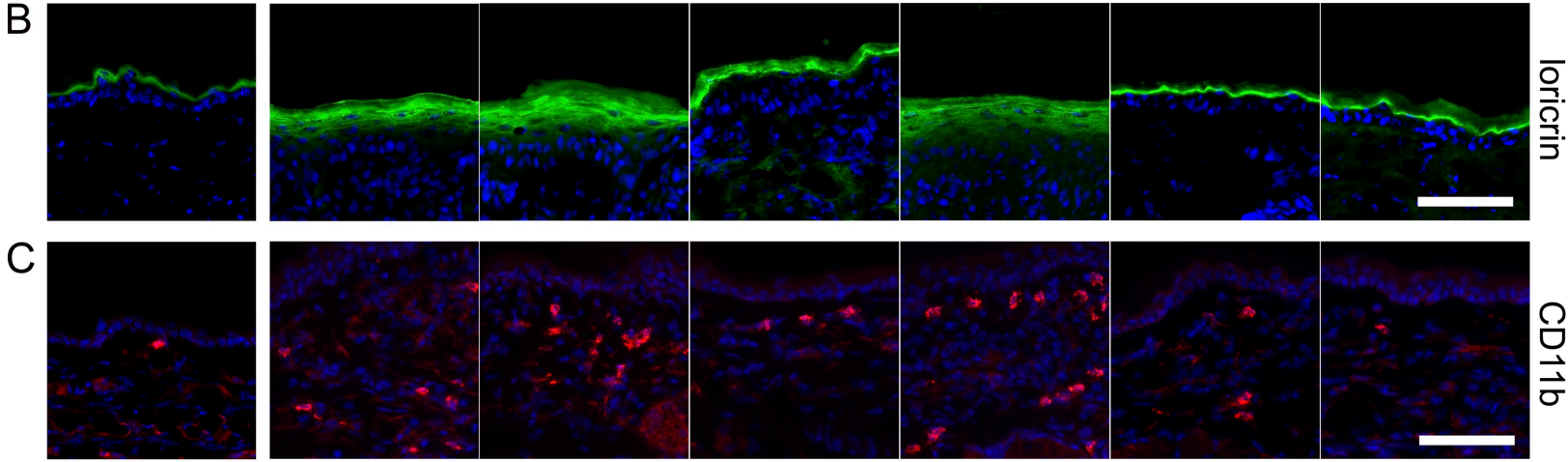

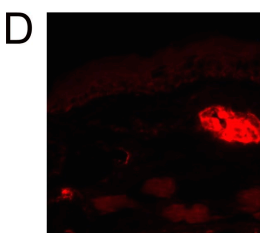

wt

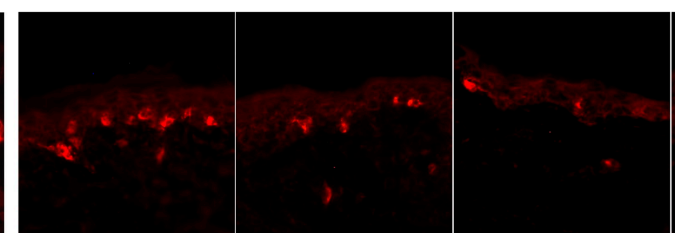

$\lg G$
anti-R1
anti-R2

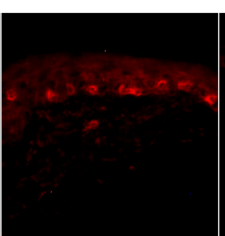

anti-R3
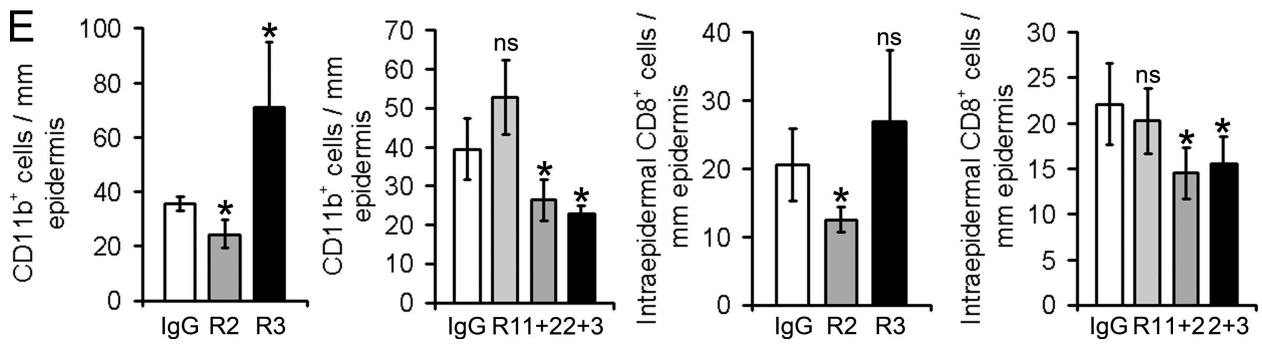

Figure 5. Systemic blockade of VEGFR-2 results in normalized epidermal differentiation and reduced inflammatory skin infiltration.

(A and B) Immunofluorescence analyses of ear skin sections from normal wild-type mice show that the hyperproliferation-associated keratin 6 is only faintly expressed in the normal interfollicular epidermis and is largely restricted to the hair follicle (A). Loricrin, a marker of terminal epidermal differentiation, is restricted to the upper granular layer in normal mouse skin (B). At day 21 of chronic skin inflammation in control lgG-treated mice, keratin 6 and loricrin show a much broader expression pattern. Inhibition of VEGFR-2 (anti-R2), alone or in combination with inhibition of VEGFR-1 or VEGFR-3, largely normalized keratin 6 and loricrin expression patterns. Treatment with anti-VEGFR-1 or anti-VEGR-3 showed no major effect. (C and E) CD11 b+ cells are rarely found in normal mouse skin (wt). The number of CD11 b+ cells was increased in the inflamed skin of control lgG-treated mice and was decreased after the inhibition of VEGFR-2, VEGFR-1+2, or VEGFR-2+3 but not after inhibition of VEGFR-1. Inhibition of VEGFR-3 resulted in enhanced numbers of CD11b+ cells. (D and E) Inhibition of VEGFR-2, VEGFR-1+2, and VEGFR-2+3 strongly decreased the number of intraepidermal CD8 ${ }^{+}$T-lymphocytes, whereas the inhibition of VEGFR-1 or VEGFR-3 had no effect, as compared with IgG-treated mice. Bars, $100 \mu \mathrm{m}$. (E) Computer-assisted quantification of the numbers of CD $11 \mathrm{~b}^{+}$and CD8 ${ }^{+}$cells per millimeter of epidermal basement membrane. $n=5$ mice per group. Two independent experiments were performed. Data represent mean \pm SD. ${ }^{*}, P<0.05$. ns, not significant.

inflamed skin, and they also suggest that lymphangiogenesis in the K14-VEGF-A mouse model of psoriasis is predominantly dependent on VEGF-A/VEGFR-2 signaling with a partial contribution of VEGF-C/VEGFR-3 signaling.

\section{Inhibition of VEGFR-2 decreases inflammatory cell} infiltration and normalizes epidermal differentiation In ear sections of normal wild-type mice, the hyperproliferation-associated keratin 6 was faintly expressed in the normal interfollicular epidermis and largely restricted to keratinocytes of the outer root sheath of the hair follicle (Fig. $5 \mathrm{~A}$ ), which is in agreement with previous studies (Stark et al., 1987). Loricrin, a marker of terminal epidermal differentiation, was restricted to the upper granular layer in uninflamed mouse skin (Fig. 5 B). In contrast, in the inflamed skin of control IgG-treated mice, keratin 6 and loricrin showed a much broader epidermal staining pattern. After systemic inhibition of VEGFR-1 or VEGFR-3, the expression pattern of keratin 6 (Fig. 5 A) and 

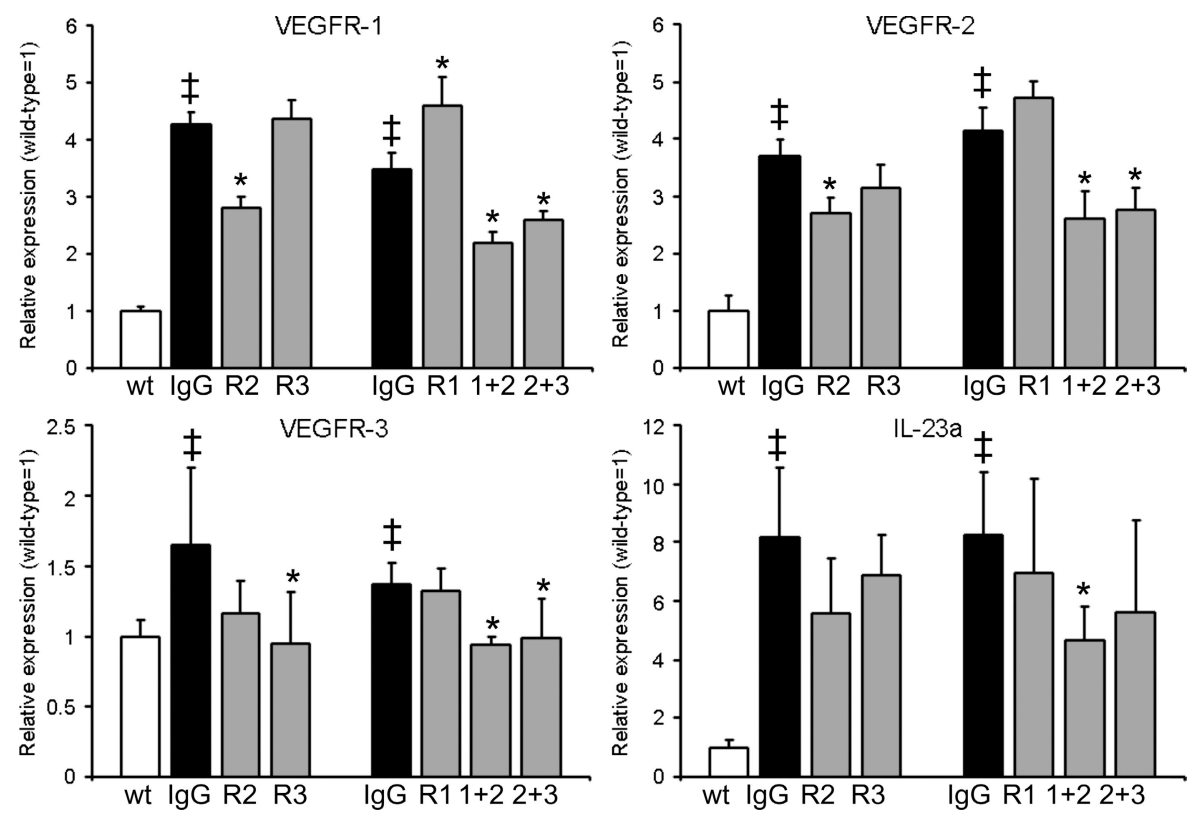

Figure 6. Expression of VEGFR and IL-23a mRNA after antibody injections. TaqMan-based real-time RT-PCR analyses were performed on whole ear skin extracts after $14 \mathrm{~d}$ of antibody treatment (study day 21) in K14-VEGF-A Tg mice. VEGFR-1, VEGFR-2, VEGFR-3, and the IL-23-specific subunit p19 (IL-23a) were significantly upregulated in inflamed IgG-injected mouse skin compared with untreated wild-type mice. The modulation of mRNA transcript levels after the corresponding antibody injections is shown. $n=5$ mice per group. Two independent experiments were performed. Data represent mean $\pm S D$. $\neq, P<0.05$ versus wild type; ${ }^{*}, \mathrm{P}<0.05$ versus IgG. R1, anti-VEGFR-1; R2, anti-VEGFR-2; R3, antiVEGFR- $3 ; 1+2$, anti-VEGFR- $1+2 ; 2+3$, antiVEGFR-2+3 antibody-injected mice.

anti-VEGFR-1+2, and anti-VEGFR$2+3 \mathrm{mAb}$ injections, resulted in signifloricrin (Fig. 5 B) remained largely unchanged. In contrast, inhibition of VEGFR-2 and, more potently, of VEGFR-1+2 or VEGFR-2+3, reverted keratin 6 and loricrin expression to a pattern seen in normal wild-type mice (Fig. 5, A and B).

$\mathrm{CD}_{11} \mathrm{~b}^{+}$cells are rarely found in normal mouse skin (Fig. $5 \mathrm{C}$ ) and $\mathrm{CD}^{+} \mathrm{T}$ lymphocytes are almost completely absent from the epidermis (Fig. 5 D). In human psoriasis and in the K14-VEGF-A Tg mouse model, there is a strong infiltration of $\mathrm{CD} 11 \mathrm{~b}^{+}$cells into the dermis and of $\mathrm{CD}^{+} \mathrm{T}$ lymphocytes into the epidermis (Fig. 5, C and D). Unexpectedly, the number of $\mathrm{CD} 11 \mathrm{~b}^{+}$cells within the inflamed skin significantly increased after inhibition of VEGFR-3. After inhibition of VEGFR-2, VEGFR-1+2, and VEGFR-2+3, the number of $\mathrm{CD}_{11} \mathrm{~b}^{+}$cells was significantly reduced, whereas inhibition of VEGFR-1 had no effect (Fig. 5, C and E). Similarly, the inhibition of VEGFR-2, VEGFR-1+2, and VEGFR-2+3 strongly decreased the number of intraepidermal $\mathrm{CD}^{+}$ T-lymphocytes, whereas the inhibition of VEGFR-1 or VEGFR-3 had no effect (Fig. 5, D and E).

We next performed real-time RT-PCR analyses of mouse ear skin extracts for the expression of all three VEGFRs after treatment for $14 \mathrm{~d}$. At study day 21, there was a significantly increased expression of VEGFR-1 (3.5-4.3fold), VEGFR-2 (3.7-4.1-fold), and VEGFR-3 (1.4-1.6-fold) in the inflamed skin of control IgG-injected VEGF-A Tg mice, as compared with uninflamed wild-type mice (Fig. 6), which is in agreement with the observed induction of inflammatory angiogenesis and lymphangiogenesis. Consistent with the lack of effects on blood vessel angiogenesis seen in the immunofluorescence analyses, anti-VEGFR-1 and antiVEGFR-3 mAb treatment did not result in a reduction of VEGFR-1 and -2 mRNA transcript levels. However, systemic inhibition of VEGFR-3 resulted in a significant down-regulation of VEGFR-3 expression. Effective antiinflammatory treatment strategies, such as anti-VEGFR-2, icant downmodulation of VEGFR-1 and VEGFR-2 mRNA expression (Fig. 6).

The IL-23- $\mathrm{T}_{\mathrm{H}} 17$ axis is important in the development of many human inflammatory diseases including psoriasis (Louten et al., 2009). It was recently shown — by a genome wide scanthat human psoriasis is associated with the IL-23 pathway (Nair et al., 2009) and that the fully human monoclonal antibody ustekinumab, which specifically inhibits the p40 subunit of the IL-12 and IL-23 cytokines, might be an effective treatment for psoriasis (Krueger et al., 2007). Using TaqMan-based realtime RT-PCR analyses, we found a strong up-regulation (8.2-8.3-fold) of the gene expression of the IL-23-specific subunit p19 (IL-23a) in the inflamed skin of IgG-injected mice, compared with uninflamed wild-type mice (Fig. 6). Specific inhibition of VEGFR-1+2 significantly decreased IL-23a expression, and anti-VEGFR-2 and anti-VEGFR-2+3 showed a tendency of reduced IL-23a expression ( $\mathrm{P}=0.057$ and 0.12 , respectively; Fig. 6). In contrast, single anti-VEGFR-1 and anti-VEGFR-3 treatments showed no effect.

\section{$\mathrm{Tg}$ overexpression of VEGF-C in the skin of VEGF-A Tg mice resolves chronic skin inflammation}

Because blockade of VEGFR-3 reduced the number of lymphatic vessels and also prolonged the course of inflammatory ear swelling, we next investigated whether enhanced cutaneous levels of the VEGFR-3 ligand VEGF-C might modulate skin inflammation. To this end, we crossed homozygous VEGF-A Tg mice with hemizygous VEGF-C Tg mice to obtain VEGF-A+C double Tg mice and single VEGF-A Tg littermates. VEGF-C Tg mice overexpress human VEGF-C under the K14 promoter and have increased numbers of lymphatic vessels in their skin with no obvious effect on the blood vessel phenotype (Jeltsch et al., 1997). Surprisingly, chronic Tg overexpression of VEGF-C in the K14-VEGF-A Tg mice had already potently reduced ear 


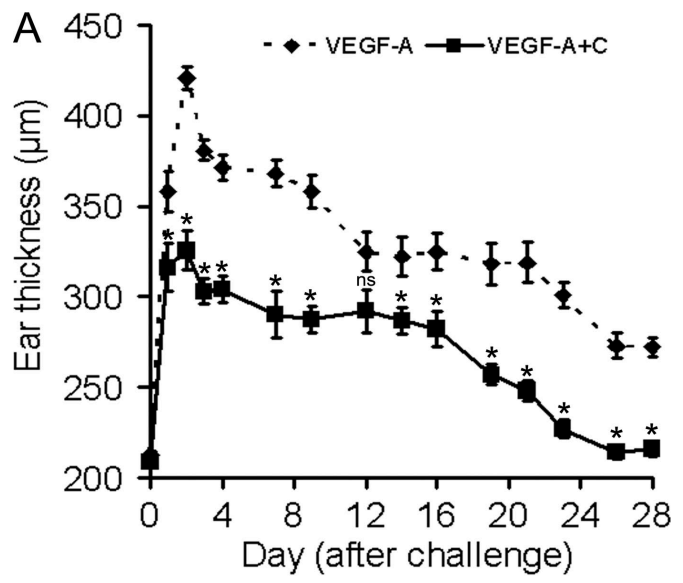

B

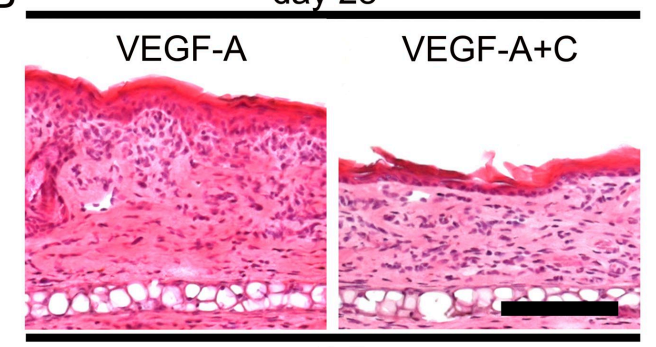

$\mathrm{H} \& \mathrm{E}$

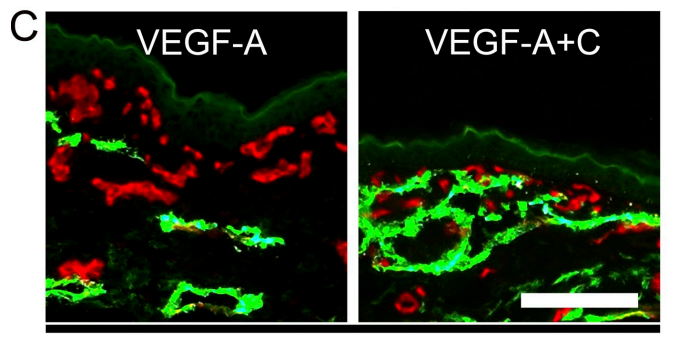

CD31+LYVE-1
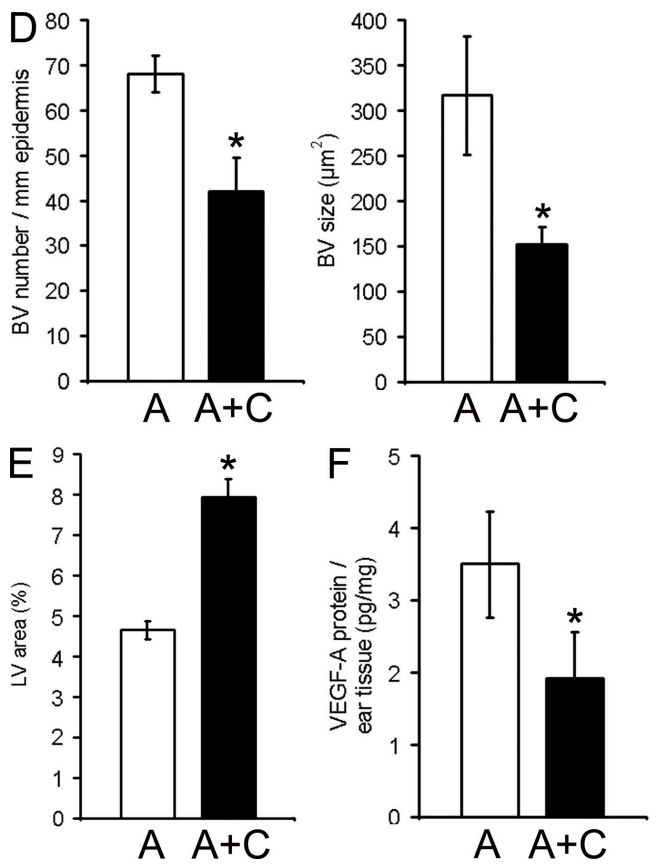

thickness $2 \mathrm{~d}$ after oxazolone challenge, which led to a complete resolution of skin inflammation by day 28 , whereas VEGF-A single Tg mice developed a chronic skin inflammation (Fig. 7 A).

Histological analyses of ear sections at day 28 revealed normalization of the skin architecture in VEGF-A +C double Tg mice (Fig. 7 B), which is similar to the uninflamed skin of control mice (Fig. 1 C), whereas VEGF-A single Tg mice showed the classical signs of epidermal hyperplasia and inflammatory cell infiltration (Fig. 7 B). Double immunofluorescence analyses of CD $31^{+} / \mathrm{LYVE}-1^{+}$lymphatic vessels showed an increased density of lymphatic vessels in the skin of VEGF-A $+\mathrm{C}$ double $\mathrm{Tg}$ mice at day 28 , as compared with VEGF-A single Tg mice (Fig. 7, C and E). Although VEGF-A single $\mathrm{Tg}$ mice showed enlarged and tortuous CD31 ${ }^{+}$LYVE-1- blood vessels, the blood vascular phenotype was largely normal in the VEGF-A $+\mathrm{C}$ double $\mathrm{Tg}$ mice (Fig. 7 C). Computer-based image analyses revealed that the blood vessel number and size (Fig. 7 D) were significantly lower in the VEGF-A+C double Tg mice than in the VEGF-A single Tg mice. In contrast, no differences were observed under noninflamed baseline conditions (Fig. S4, A and B).

The VEGF-A level is a good marker for inflammation in human psoriasis and in the K14-VEGF-A mouse model of chronic skin inflammation (Bhushan et al., 1999; Kunstfeld et al., 2004; Halin et al., 2007, 2008). In agreement with an overall improvement of inflammation, VEGF-A protein levels were significantly lower $(\mathrm{P}<0.05)$ in VEGF-A+C mice than in VEGF-A Tg mice (Fig. 7 F) at day 28, whereas the VEGF-A protein levels were similar in the normal/nonpsoriatic skin of both types of mice $(5.1 \pm 1.0 \mathrm{pg} / \mathrm{mg}$ for VEGF-A vs. $4.8 \pm 0.6 \mathrm{pg} / \mathrm{mg}$ for VEGF-A+C Tg mice; $\mathrm{P}>0.05)$.

Figure 7. Chronic Tg overexpression of VEGF-C in the skin of K14-VEGF-A Tg mice prevents chronic skin inflammation.

(A) K14-VEGF-A Tg mice (VEGF-A; $n=5$ ) and K14-VEGF-A+C double Tg mice (VEGF-A+C; $n=5$ ) were painted and challenged using oxazolone as described in Fig. 1. Ear thickness was measured over $28 \mathrm{~d}$. Inflammatory ear swelling was significantly reduced in VEGF-A+C double Tg mice, as compared with VEGF-A Tg mice. Importantly, ear thickness of VEGF-A+C Tg mice went back to normal levels within 4 wk. Data represent mean \pm SEM. (B) H\&E-stained sections revealed reduced edema formation, epidermal thickening, and inflammatory cell infiltration in K14-VEGF-A+C double Tg mice as compared with K14-VEGF-A mice at day 28 after oxazolone challenge. (C) At day 28, differential immunofluorescence stains for lymphatic vessels (CD31+/LYVE-1+; green) and blood vessels (CD31+/LYVE-1-; red) revealed that K14-VEGF-A+C mice have a strong increase in their lymphatic vessel network compared with K14-VEGF-A Tg mice. Bars, $100 \mu \mathrm{m}$. ( $D$ and $E$ ) At day 28 after oxazolone challenge, computer-assisted image analyses revealed a significantly reduced number and mean size of blood vessels (BV; D) and an increased density of lymphatic vessels (LV; E) in the K14-VEGF-A+C $(A+C)$ double Tg mice compared with K14-VEGF-A (A) Tg mice. (F) ELISA analyses of ear lysates showed decreased levels of VEGF-A in double Tg K14-VEGF-A+C mice compared with K14-VEGF-A Tg mice at $28 \mathrm{~d}$ after oxazolone challenge. Two independent experiments were performed. Data represent mean $\pm S D .{ }^{*}, P<0.05$. ns, not significant. 
A

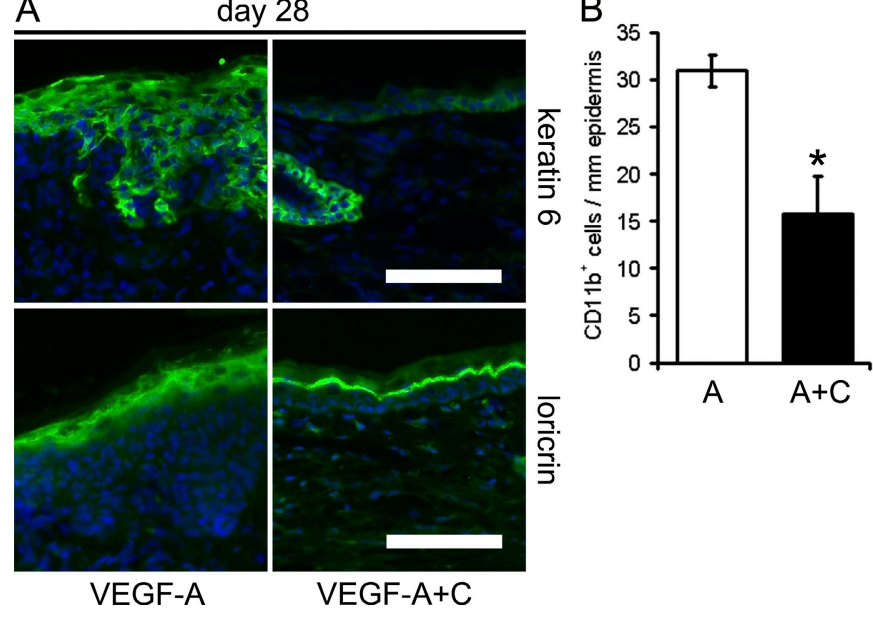

Genetic overexpression of VEGF-C in the skin of VEGF-A Tg mice decreases inflammatory cell infiltration and normalizes epidermal architecture

At day 28, the hyperproliferation-associated marker keratin 6 was almost absent in the epidermis of VEGF-A $+\mathrm{C}$ double $\mathrm{Tg}$ mice and restricted to the hair follicle, similar to the findings in normal uninflamed wild-type mice, but was still present in the VEGF-A single Tg mice (Fig. $8 \mathrm{~A}$ and Fig. 5 A). The expression of the differentiation marker loricrin was restricted to the upper granular layer in the VEGF-A+C double $\mathrm{Tg}$ mice but not in VEGF-A single Tg mice, with a pattern very similar to that of uninflamed wild-type mice (Fig. $8 \mathrm{~A}$ and Fig. 5 B). Importantly, there was no baseline difference under noninflamed conditions (Fig. S4 A).

Genetic overexpression of VEGF-C in the skin of VEGF-A Tg mice also strongly reduced the number of CD11 ${ }^{+}$monocytes/granulocytes in the dermis and of $\mathrm{CD}^{+} \mathrm{T}$-lymphocytes in the epidermis, as compared with VEGF-A single Tg mice (Fig. 8, B and C). Using a quantitative cytokine array, our preliminary data indicate that the protein levels of IL-17, IL-1 $\beta$, M-CSF, and MCP-1 were significantly lower in the double Tg VEGF-A +C mice than in single VEGF-A Tg mice. TNF, IFN- $\gamma$, IL-12, and IL-1 $\alpha$ levels were also lower in the skin of VEGF-A $+\mathrm{C}$ double Tg mice than in VEGF-A Tg mice (Fig. S3).

\section{Chronic skin inflammation in K14-VEGF-A mice is associated with impaired lymphatic function}

Because K14-VEGF-A+C double Tg mice were characterized by reduced skin inflammation, we next asked if dysfunction of lymphatic vessels might contribute to the chronic skin inflammation in the K14-VEGF-A mouse model. We performed in vivo near-infrared imaging of lymphatic drainage using the IVIS system. We injected indocyanine green-containing liposomes into the ear skin of K14-VEGF-A Tg mice and assessed the fluorescent signal in the draining superficial parotid lymph node (Van den Broeck et al., 2006) over a time course of 25 min. The positioning of the mice, a representative image of a fluorescently labeled lymph node, and the data analysis are shown in

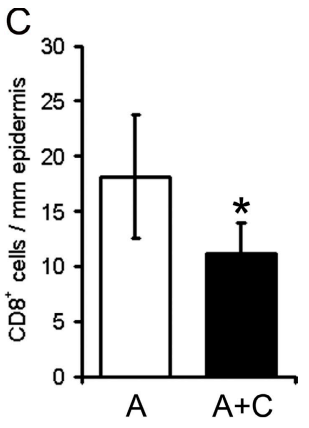

Figure 8. Tg overexpression of VEGF-C in the skin of K14-VEGF-A Tg mice reduces inflammatory cell infiltration and normalizes epidermal proliferation and differentiation. (A) Immunofluorescence analyses revealed that the hyperproliferation marker keratin 6 was restricted to hair follicles, and that the differentiation marker loricrin was restricted to the suprabasal layers of the epidermis in the K14-VEGF-A+C Tg mice, whereas both keratin 6 and loricrin were broadly expressed in the epidermis of K14-VEGF-A Tg mice. Bars, $100 \mu \mathrm{m}$.

( $B$ and $C$ ) Quantitative image analyses of ear skin sections at study day 28 revealed significantly reduced numbers of dermal CD11 $\mathrm{b}^{+}$ monocytes/granulocytes (B) and intraepidermal CD8 ${ }^{+} T$ cells (C) in K14-VEGF-A+C double $\mathrm{Tg}$ mice compared with K14-VEGF-A single Tg

Fig. S5 and Fig. S6. 2 d after oxazolone challenge, the mice. $n=5$ per group. Two independent experiments were performed. Data represent mean \pm SD. ${ }^{*}, \mathrm{P}<0.05$. the lymph node to efferent lymphatic vessels was similar to the uninflamed state, as indicated by the half-life time of dye fluorescence intensity in the lymph node $(4.42 \pm 1.95$ vs. $2.78 \pm 0.55$ min; $\mathrm{P}>0.05$; Fig. 9 A). The lymph flow was decreased at day 7 and was significantly reduced at day 14 after oxazolone challenge, as compared with uninflamed K14-VEGF-A Tg mice, indicating dysfunction of the lymphatic vasculature during chronic skin inflammation (Fig. 9 A). Importantly, the lymph flow was significantly enhanced in the K14-VEGF-A+C double Tg mice at day 14 after induction of inflammation, as compared with the K14-VEGF-A single Tg mice (Fig. 9 B). These data suggest that increased VEGF-C-induced lymphatic flow might contribute to resolving chronic skin inflammation in the K14VEGF-A+C double Tg mice.

To further investigate whether the antiinflammatory effect of $\mathrm{Tg}$ overexpression of VEGF-C (which might bind both VEGFR-3 and VEGFR-2) was indeed mediated via VEGFR-3 activation on lymphatic endothelium in the skin, we next established a new Tg mouse model with chronic Tg overexpression of mouse VEGF-D in the skin of K14-VEGF-A Tg mice. In contrast to VEGF-C, mouse VEGF-D is a specific ligand for VEGFR-3 but not for VEGFR-2 (Baldwin et al., 2001). Sensitization and challenge with oxazolone of K14-VEGF-A single $\mathrm{Tg}$ mice resulted in chronic skin inflammation, whereas K14-VEGF-A+D double Tg mice showed a significantly reduced ear thickness, similar to what we observed in the K14VEGF-A+C double Tg mice (Fig. 10 A).

VEGF-A/-C and VEGF-A/-D double Tg mice show chronically elevated levels of VEGF-C or VEGF-D in the skin and, thus, an increase in the number of preexisting lymphatic vessels before the induction of inflammation might have contributed to the inhibition of chronic inflammation. Thus, we next investigated whether acute delivery of VEGF-C to the skin might also ameliorate cutaneous inflammation. 

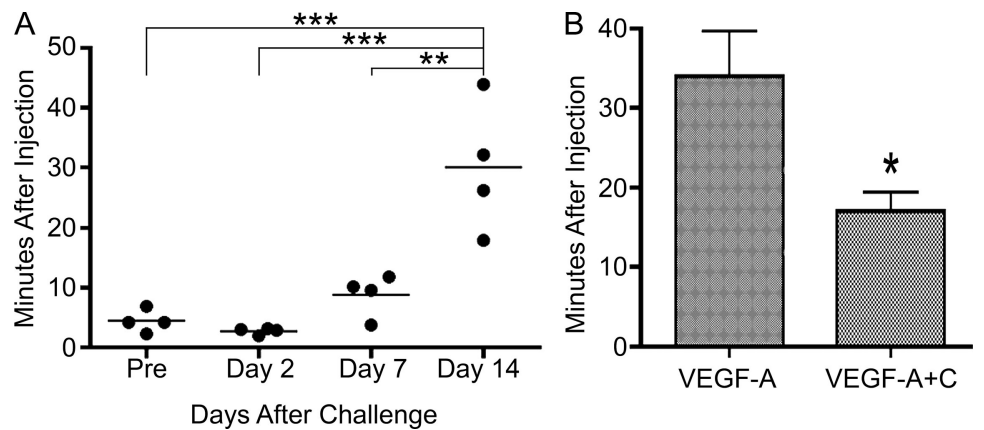

Figure 9. Lymphatic vessel drainage function is impaired during chronic skin inflammation and is enhanced by $\mathrm{Tg}$ overexpression of VEGF-C. (A) K14-VEGF-A Tg mice $(n=4)$ were sensitized and challenged using oxazolone. Indocyanine green-containing liposomes were injected intradermally into the ear skin, and the lymphatic drainage from the superficial parotid lymph node was monitored in vivo using the Xenogen IVIS system over a period of $25 \mathrm{~min}$. The lymph flow was unaltered at $2 \mathrm{~d}$ after induction of skin inflammation, as compared with normal skin (Pre), but progressively declined until day 14 , as indicated by the significantly increased half-life time. An exponential decay model was used to calculate half-life times. Two independent experiments were performed. Horizontal bars indicate mean.

${ }^{*}, \mathrm{P}<0.05 ;{ }^{* *}, \mathrm{P}<0.01 ;{ }^{* * *}, \mathrm{P}<0.001$. (B) K14-VEGF-A $(n=4)$ and K14-VEGF-A+C $(n=4)$ Tg mice were treated as described in A, and indocyanine greencontaining liposomes were injected intradermally at $14 \mathrm{~d}$ after oxazolone challenge. The lymphatic flow from the lymph node was significantly higher in the K14-VEGF-A+C double Tg mice than in the K14-VEGF-A single Tg mice, as indicated by the significantly shorter half-life time. Two independent experiments were performed. Data represent mean $\pm S D$. ${ }^{*}, P<0.05$.

In particular, we were interested whether specific activation of VEGFR-3 might inhibit inflammation. To this end, we injected recombinant VEGF-C156S protein, a VEGFR-3specific mutant which does not activate VEGFR-2 (Joukov et al., 1998), daily into the inflamed ear skin of K14-VEGF-A Tg mice during the chronic phase of skin inflammation, starting at day 7 after oxazolone challenge. Already after $5 \mathrm{~d}$ of VEGF-C156S therapy, ear swelling was significantly reduced, as compared with PBS-injected controls (Fig. 10 B). The antiinflammatory effect was maintained until the end of the treatment at day 21 (Fig. 10 B). Although there were no major changes of lymph flow (unpublished data), histological analyses revealed a significantly reduced blood vessel size ( $282 \pm 44$ vs. $\left.373 \pm 82 \mu \mathrm{m}^{2} ; \mathrm{P}<0.05\right)$, increased lymphatic vessel size (1064 \pm 303 vs. $\left.677 \pm 169 \mu \mathrm{m}^{2} ; \mathrm{P}<0.05\right)$, and increased area covered by lymphatic vessels (11.8 \pm 2.8 vs. $6.6 \pm 0.7 \%$; $\mathrm{P}<0.05)$ after injection of VEGFC156S as compared with PBS injection. The number of blood or lymphatic vessels was not different between the two groups. Furthermore, the number of $\mathrm{CD} 11 \mathrm{~b}^{+}$cells was significantly decreased after intradermal injections of VEGF-C156S as compared with PBS (39 \pm 9 [VEGFC156S] vs. $63 \pm 10$ [PBS] cells $/ \mathrm{mm}$ epidermal basement membrane; $\mathrm{P}<0.05)$.

\section{DISCUSSION}

In this paper, we have investigated the individual role of the three VEGFRs and of lymphatic vessels in a VEGF-A-driven mouse model of chronic skin inflammation. To this end, we used pharmacological approaches to analyze the effect of specific inhibition of VEGFR signaling on chronic skin inflammation and we also applied genetic approaches by establishing new mouse models for the chronic Tg delivery of both VEGF-C and VEGF-A, or of both mouse VEGF-D and VEGF-A, under control of the K14 promoter. We found that systemic inhibition of angiogenesis by blockade of VEGFR-2 significantly ameliorated the course of cutaneous inflammation, whereas inhibition of VEGFR-3 prolonged inflammatory edema formation despite inhibition of lymphangiogenesis. To our knowledge, this is the first study which shows that specific activation of lymphatic vessels by $\mathrm{Tg}$ overexpression of VEGF-C or the VEGFR-3-specific ligand mVEGF-D, or by the injection of the VEGFR-3-specific mutant VEGFC156S, inhibits chronic inflammation.

Most of the major parameters of psoriasis skin lesions, including epidermal hyperproliferation and impaired differentiation, pathological angiogenesis, inflammatory cell infiltration with accumulation of CD4 cells in the dermis and CD8 cells in the epidermis, and the expression of IL-23a,

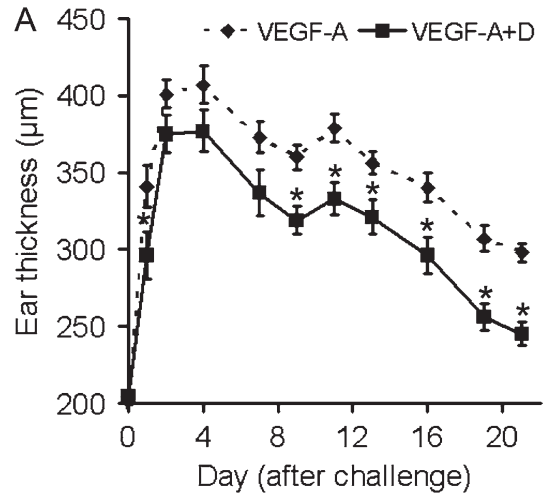

2264
Figure 10. Specific activation of VEGFR-3 reduces inflammatory ear swelling in the K14-VEGF-A mouse model. (A) Hemizygous K14-VEGF-A+D $(\boldsymbol{\square}, n=5)$ and K14VEGF-A $(\bullet, n=5)$ Tg mice were painted at day -5 with $2 \%$ oxazolone and challenged at day 0 with $1 \%$ oxazolone on the ear skin. (B) K14-VEGF-A Tg mice $(n=11)$ were treated as described in A. Starting at study day 7 , the mice received daily intradermal injections of $200 \mathrm{ng}$ of recombinant VEGF-C156S protein $(O, n=6)$, a VEGFR-3-specific mutant, or of $\operatorname{PBS}(\boldsymbol{\Lambda}, n=5)$ for $14 \mathrm{~d}$. Two independent experiments were performed. Data represent mean \pm SEM. ${ }^{*}, \mathrm{P}<0.05$. 
were also found in the K14/VEGF-A Tg model and were largely normalized after specific inhibition of angiogenesis or specific stimulation of lymphangiogenesis. Importantly, we found that the chronic inflammation in the K14-VEGF-A mouse model is accompanied by impaired lymphatic vessel function and that $\mathrm{Tg}$ delivery of VEGF-C to the skin restores the lymphatic vessel function in this model. Together, these findings surprisingly indicate that stimulation of lymphangiogenesis and reversion of lymphatic vessel dysfunction, which is primarily mediated through VEGF-A in this model system (Kajiya et al., 2006), might be sufficient to resolve chronic skin inflammation.

The receptor tyrosine kinase VEGFR-2 is thought to be the main mediator of VEGF-A-driven endothelial cell proliferation, differentiation, and sprouting (Adams and Alitalo, 2007). This is consistent with our data showing potent antiangiogenic effects after blockade of VEGFR-2 in chronic inflammation induced in K14/VEGF-A Tg mice. In contrast, short-term inhibition of VEGFR-2 in an acute delayed-type hypersensitivity model in wild-type mice did not ameliorate acute skin inflammation (Kunstfeld et al., 2004).

The expression of VEGFR-3 has been generally thought to be restricted to the lymphatic vascular system, with some exceptions (Partanen et al., 2000; Hamrah et al., 2003), and the biological effects of inhibition of VEGFR-3 have been attributed to its effects on activated lymphatic vessels (Pytowski et al., 2005). Recently, it has been reported that VEGF-A induces the expression of VEGFR-3 on angiogenic sprouts during embryonic development and also in distinct pathological settings (Tammela et al., 2008). Our current findings reveal that in the setting of chronic skin inflammation in mice, the tortuous cutaneous blood vessels, in the vicinity of the VEGF-Asecreting epidermis, lack expression of VEGFR-3, as compared with the strong VEGFR-3 expression detected on MECA32-negative lymphatic vessels. These findings likely explain why we did not detect any major effects of VEGFR-3 blockade on the cutaneous blood vasculature, as assessed histologically and by measuring the expression levels of all three VEGFRs. These findings also indicate that specific activation of VEGFR-3 in inflamed skin should not promote skin angiogenesis. Indeed, we did not find any further induction of blood vessel angiogenesis in the skin of VEGF-A/VEGF-D double Tg mice, as compared with VEGF-A single Tg mice.

Although the single inhibition of VEGFR-1 did not ameliorate inflammation or angiogenesis, and VEGFR-3 blockade prolonged inflammation, the combined blockade of VEGFR$1+2$ and VEGFR-2+3 showed even more pronounced inhibitory effects than the single inhibition of VEGFR-2 on all parameters analyzed. This might be explained by a role of heterodimerization of the VEGFRs in modulating the signaling cascade. VEGF-E, which specifically activates VEGFR-2, induces angiogenesis without signs of edema and hemorrhaging (Kiba et al., 2003). This finding shows that VEGFR-1/-2 cross talk might be required for driving vascular permeability. Additionally, heterodimerization of VEGFR-3 with VEGFR-2 results in a different use of phosphorylation sites upon ligand binding compared with the homodimeric receptor situation (Dixelius et al., 2003).

Inflammation-induced lymphangiogenesis can be regulated by direct VEGF-A/VEGFR-2 and by VEGF-C/VEGF-D/ VEGFR-3 signaling and might be modulated by the attraction of inflammatory cells releasing lymphangiogenic factors (Baluk et al., 2005; Kataru et al., 2009; Wuest and Carr, 2010). However, VEGF-A-induced lymphatic vessels might be less functional than those induced by VEGF-C or VEGF-D/ VEGFR-3 signaling (Nagy et al., 2002; Kajiya et al., 2006), although one has to keep in mind that mouse VEGF- $\mathrm{A}_{164}$ and the human isoform VEGF- $\mathrm{A}_{165}$ likely transduce different signals in lymphatic vessels in vivo (Wirzenius et al., 2007). Our findings indicate that lymphangiogenesis in the K14VEGF-A mouse model of psoriasis is mainly dependent on VEGFR-2 but that VEGFR-3 signaling partially participates in driving lymphangiogenesis. This is mainly caused by the lymphangiogenic factor VEGF-C, which was up-regulated during chronic skin inflammation. Interestingly, a recent publication on HSV-1-infected cells found that VEGF-A is the sole inductor of corneal lymphangiogenesis (Wuest and Carr, 2010).

Overall, our VEGFR blocking studies revealed that inhibition of angiogenesis is sufficient to resolve chronic skin inflammation. In contrast, specifically reducing inflammatory lymphangiogenesis by inhibition of VEGFR-3 delayed edema resolution. It is likely that the increased edema observed after specific blockade of VEGFR-3 is a result of improper lymphatic drainage. It has recently been shown that the inhibition of VEGF-C/-D by sVEGFR-3 significantly decreased lymph flow in a model of bacterial skin inflammation (Kataru et al., 2009), that genetic overexpression of soluble VEGFR-3 in the skin of mice resulted in a lymphedema-like phenotype (Mäkinen et al., 2001a), and that the inhibition of VEGFR-3 decreased lymph flow in a mouse model of chronic inflammatory arthritis (Guo et al., 2009). Importantly, both of the latter models are strongly TNF dependent. Conversely, application of VEGF-C or tumor-derived VEGF-C have been found to enhance lymphatic function and flow, respectively (Karkkainen et al., 2001; Szuba et al., 2002; Yoon et al., 2003; Hoshida et al., 2006; Tammela et al., 2007). The impaired lymphatic drainage might also explain the accumulation of CD11 $\mathrm{b}^{+}$ cells that we observed after blockade of VEGFR-3. Interestingly, the $\mathrm{CD}_{11 \mathrm{~b}^{+}}$cells in the skin lacked the expression of VEGFR-3, unlike the CD11 $\mathrm{b}^{+}$cells in the inflamed cornea which have been reported to uniformly express VEGFR-3 (Hamrah et al., 2004).

Our study reveals that the lymphatic vessels become dysfunctional during the establishment of chronic inflammatory skin lesions and that lymphatic function can be restored by genetically overexpressing VEGF-C. We assessed the lymph flow at study day 14, when the skin inflammation in the K14-VEGF-A mouse model reaches the most chronic state and when ear thickness remains largely constant (Fig. 7 A). We unexpectedly found that the half-life time of dye disappearance from the ear-draining superficial parotid lymph 
node was increased as compared with the preinflammatory and acute inflammatory state (Fig. 9 A), revealing that the lymphatic drainage function is impaired in chronic inflammation. Inflammation-induced lymphangiogenesis might therefore represent an endogenous counter-regulatory mechanism aimed at limiting edema formation and accumulation of inflammatory cells, and therapeutic application of VEGF-C might further promote this mechanism. Nevertheless, we cannot fully exclude that other VEGF-C activities, including other direct effects on the inflamed lymphatic vasculature, are involved in its antiinflammatory properties. As an example, the chemokine receptor D6 is expressed on lymphatic vessels and sequesters several proinflammatory chemokines. D6-deficient mice show histological alterations similar to human psoriasis after treatment with phorbol esters (Jamieson et al., 2005). An increased amount of D6 receptor, caused by an overall increase in the lymphatic vasculature after VEGFR-3 activation, might contribute to the strong improvement of the chronic inflammatory skin lesions. That there was no further increase in measured flow after injection of VEGF-C156S might be the result of a limitation of the near-infrared imaging approach, which does not specifically measure lymph flow from the ear to the draining lymph node. Additionally, constant repetitive injections into the ear skin, causing local irritations, might disturb flow measurements where dye is injected into the same ear. Fig. S7 shows a schematic diagram of the proposed model.

Although not assessed in this study, it would be of interest to investigate the potential role of lymph node lymphangiogenesis in the control of inflammation (Angeli et al., 2006; Halin et al., 2007). The increased draining capacity and resolution of chronic skin inflammation might be, at least in part, mediated by enhanced lymph node lymphangiogenesis in addition to the increased lymphangiogenesis that we observed in the lesional skin itself. In conclusion, our study provides the first proof of concept that it might be possible to treat chronic inflammatory skin disorders by increasing lymphangiogenesis and stimulating lymphatic vessel function, with potential implications for the treatment of other chronic inflammatory diseases.

\section{MATERIALS AND METHODS}

Mouse model of chronic skin inflammation. The generation of K14VEGF-A Tg mice that express mouse VEGF-A164 under control of the K14 promoter, of K14-VEGF-C Tg mice that express human VEGF-C, and of K14-VEGF-D Tg mice that express mouse VEGF-D has been described previously (Jeltsch et al., 1997; Detmar et al., 1998; Xia et al., 2003; Haiko et al., 2008). K14-VEGF-A, K14-VEGF-C, and K14-VEGF-D Tg mice (all FVB genetic background) were bred and housed in the animal facility of ETH Zurich. K14-VEGF-A+C and K14-VEGF-A+D double Tg mice were generated by crossing homozygous VEGF-A and hemizygous VEGF-C or VEGF-D Tg mice, respectively. The mice were genotyped as previously described (Jeltsch et al., 1997; Haiko et al., 2008). Untreated FVB wild-type mice were used as controls. All experiments were initiated when the mice were between 6 and 8 wk of age. Experiments were performed in accordance with animal protocol 149/2008 approved by the Kantonales Veterinäramt Zürich.

For oxazolone-induced psoriasis-like skin inflammation in the ear skin (Kunstfeld et al., 2004), mice were anesthetized by i.p. injection of $0.2 \mathrm{mg} / \mathrm{kg}$ medetomidine and $80 \mathrm{mg} / \mathrm{kg}$ ketamine, and $2 \%$ oxazolone (4-ethoxymethylene-2 phenyl-2-oxazoline-5-one; Sigma-Aldrich) in acetone/olive oil $(4: 1 \mathrm{vol} / \mathrm{vol})$ was applied topically to the shaved abdomen $(50 \mu \mathrm{l})$ and to each paw $(5 \mu \mathrm{l}) .5 \mathrm{~d}$ after sensitization (day 0$)$, both ears were challenged by topical application of $10 \mu \mathrm{l}$ oxazolone (1\%) on each side. Ear thickness was measured before challenge and repeatedly after challenge using calipers. Total ear thickness was measured as readout of inflammation. Starting on day $7,800 \mu \mathrm{g}$ of a blocking rat anti-mouse VEGFR-1 mAb (MF1), rat anti-mouse VEGFR-2 (DC101), rat anti-mouse VEGFR-3 mAb (mF431C1; all from Imclone Systems Inc.), $800 \mu \mathrm{g}$ each of MF1 + DC101, $800 \mu \mathrm{g}$ each of DC101 + mF4-31C1, or control rat IgG (Sigma-Aldrich) was administered i.p. to K14-VEGF-A Tg mice for 14 d every third day $(n=5$ per group). This dosage was previously shown to efficiently inhibit VEGFR signaling in vivo (Baluk et al., 2005). On day 21, mice were injected with $300 \mu \mathrm{l}$ PBS containing $40 \mathrm{mM}$ BrdU (Sigma-Aldrich). After $2.5 \mathrm{~h}$, mice were killed and one ear was embedded in optimal cutting temperature (OCT) compound (Sakura). The other ear was stored in RNALater solution (Applied Biosystems).

In additional experiments, K14-VEGF-A+C $(n=9)$ and K14-VEGF$\mathrm{A}+\mathrm{D}(n=5)$ double Tg mice and their single Tg K14-VEGF-A littermates $(n=14)$ were treated with oxazolone as indicated in the previous paragraph. The ears were analyzed at study day 28. All experiments were performed at least twice.

In addition, hemizygous K14-VEGF-A Tg mice were treated for $2 \mathrm{wk}$, starting on study day 7 (after oxazolone challenge) by daily intradermal injections (into the ear skin) with 200 ng of recombinant VEGF-C (Cys156Ser; R\&D Systems) dissolved in $1 \mu \mathrm{l}$ PBS $(n=6)$ using a Hamilton syringe. The control group was injected with PBS alone $(n=5)$.

Immunohistology. On study day 21 or 28 , respectively, mice were killed and their ears were collected. Tissues were embedded in OCT compound, frozen on dry ice, and 7- $\mu \mathrm{m}$ cryostat sections were cut. Specimens were placed on glass slides, air dried, and fixed with acetone for $2 \mathrm{~min}$ at $-20^{\circ} \mathrm{C}$. After rehydration with $80 \%$ methanol at $4^{\circ} \mathrm{C}$, PBS, and PBS with $12 \%$ BSA, the specimens were incubated with the respective antibodies. Standard H\&E stainings were performed, and immunofluorescence was performed as previously described (Kunstfeld et al., 2004; Halin et al., 2008) using the following antibodies: anti-mouse LYVE-1 (AngioBio), biotin anti-MECA-32, anti-mouse CD31 (both from BD), anti-BrdU-Alexa Fluor 594 (Invitrogen), anti-mouse VEGFR-2 (AF644), anti-mouse VEGFR-3 (AF743; both R\&D Systems), anti-mouse keratin 6, loricrin (both from Covance Research Products), biotin anti-mouse CD11b, and CD8 (BD). Cy3-conjugated Streptavidin was purchased from Rockland Immunochemicals, Inc., and Alexa Fluor 488- and Alexa Fluor 594-coupled secondary antibodies and Hoechst 33342 were purchased from Invitrogen.

Computer-assisted morphometric analyses. Double immunofluorescence stains of ear sections for MECA-32, CD31, and/or LYVE-1 were examined on an Axioskop 2 mot plus microscope (Carl Zeiss, Inc.), equipped with an AxioCam MRc camera and a Plan-APOCHROMAT $10 \times / 0.45$ NA objective (Carl Zeiss, Inc.). Images of three to four individual fields of view were acquired per section using AxioVision software 4.7.1 (Carl Zeiss, Inc.). Computer-assisted analyses of digital images were performed using the IP-LABORATORY software (Scanalytics) as previously described (Schonthaler et al., 2009). The mean lymphatic and blood vessel number per millimeter of epidermal basement membrane and the mean size of $\mathrm{CD} 31^{+} / \mathrm{LYVE}_{-} 1^{+}$lymphatic and of CD $31^{+} / \mathrm{LYVE}^{-} 1^{-}$or MECA-32 ${ }^{+}$ blood vessels were determined in the area of one ear half between cartilage and stratum corneum. The results are expressed as vessel number per millimeter of epidermal basement membrane (excluding follicular structures) and not as vessel number per area because the formation of inflammatory edema (increase in area) would confound the vessel number if it were calculated per area. To quantify $\mathrm{CD} 11 \mathrm{~b}^{+}, \mathrm{CD}^{+}$, and $\mathrm{BrdU}^{+}$cell numbers per millimeter of epidermal basement membrane, images of three to four individual fields of view were acquired per sample (covering the entire field of view between the cartilage backbone and the epidermis). 
Quantitative real-time RT-PCR. Total cellular RNA was isolated from mouse ears using a TissueLyser, stainless steel beads, and the RNeasy Mini kit (all from QIAGEN) and was treated with RQ1 RNase-free DNase (Promega). $1 \mu \mathrm{g}$ RNA was used to synthesize cDNA using the High-Capacity cDNA Reverse Transcription kit (Applied Biosystems). The expression of mouse VEGF-C, VEGF-D, VEGFR-1, VEGFR-2, VEGFR-3, and IL-23a was investigated by TaqMan real-time RT-PCR using the AB 7900 HT Fast Real-Time PCR System (Applied Biosystems) and quantified using the $2^{-\Delta \Delta C T}$ method (Schmittgen and Livak, 2008). The probes and primers for VEGF-C (Mm01202432_m1), VEGF-D (Mm00438965_m1), VEGFR-1 (Mm00438971_m1), VEGFR-2 (Mm00440111_m1), VEGFR-3 (Mm00433337_m1), and IL-23a (Mm01160011_g1) were predesigned. Each reaction was multiplexed with $\beta$-actin (available from GenBank/ EMBL/DDBJ under accession no. NM_007393.3; all Applied Biosystems) as a reference gene and all data were normalized based on the expression levels of $\beta$-actin. $n=5$ per group.

ELISA for VEGF-A. Skin lysates were obtained from ear skin of VEGF-A and VEGF-A $+\mathrm{C}$ Tg mice $28 \mathrm{~d}$ after challenge with oxazolone $(n=5$ per group) and of untreated VEGF-A and VEGF-A+C Tg mice $(n=6$ per group). Tissues were homogenized in lysis buffer $(150 \mathrm{mM} \mathrm{NaCl}, 50 \mathrm{mM}$ Tris, $\mathrm{pH} 7.5$, with a protease inhibitor cocktail [Roche]). Homogenates were centrifuged for $10 \mathrm{~min}$ at $14,000 \mathrm{~g}$. Supernatants were assayed using a VEGF-A ELISA (Quantikine; R\&D Systems). The absorbance was measured with an Infinite M200 microplate reader (Tecan). VEGF-A levels were normalized to milligram of tissue.

Quantitative cytokine array. The cytokine levels in the ear skin of K14VEGF-A and K14-VEGF-A+C double Tg mice were measured quantitatively on study day 28 (after oxazolone challenge) using the Quantibody Mouse Cytokine Array 1 (RayBiotech) according to the manufacturer's instructions. The signal was visualized using GenePix Professional 4200A (Bucher Biotec AG). Data extraction was done using GenePix Pro 5.1 software. Cytokine levels were normalized to milligram of tissue.

Near-infrared imaging of lymph flow. K14-VEGF-A $(n=4)$ and K14VEGF-A $+\mathrm{C}(n=4)$ mice were anesthetized and carefully shaved in the region of the draining superficial parotid lymph node (Van den Broeck et al., 2006). During imaging, the mice were anesthetized with $2.5 \%$ isoflurane and were positioned in an IVIS Imaging System (Xenogen Corp). Using a Hamilton syringe, $3 \mu \mathrm{l}$ of indocyanine green-containing liposomes were injected intradermally into the mouse ear. The fluorescent signal of the superficial parotid lymph node was monitored over 25 min every $30 \mathrm{~s}$, using an infrared laser and Living Image software (Xenogen). This measurement is indicative of the lymph flow from the lymph node to efferent lymphatic vessels. All illumination settings were identical between the mice. The half-life of the dye fluorescence intensity in the draining lymph node was calculated by fitting an exponential decay model.

Statistics. Statistical analysis was performed using Prism version 4.03 (GraphPad Software, Inc.) or SPSS 16.0. Data are shown as mean \pm SD or \pm SEM as indicated and were analyzed with a two-tailed unpaired Student's $t$-test. When more than two groups were compared, ANOVA was applied and the individual groups were compared using a Tukey-HSD post-hoc test. Homogeneity of variances was assessed using Levene's test, and normalized distribution was assessed using Q-Q plots. Differences were considered statistically significant when $\mathrm{P}<0.05$.

Online supplemental material. Fig. S1 shows the blood vessel analysis in K14-VEGF-A Tg mice after the injection of VEGFR-blocking antibodies. Fig. S2 demonstrates the presence of VEGFR-2 on normal and inflamed lymphatic vessels. Fig. S3 shows protein levels in the ear skin of inflamed K14-VEGF-A versus K14-VEGF-A+C Tg mice. Fig. S4 shows that there is no baseline difference in vascularity between K14-VEGF-A and K14VEGF-A+C Tg mice. Fig. S5 and Fig. S6 show representative data curves and the fluorescence signal in the lymph node using the near-infrared imaging technique. Fig. S7 shows a schematic diagram of our proposed model of inhibition of chronic inflammation by stimulation of lymphangiogenesis via VEGFR 3. Online supplemental material is available at http://www.jem .org/cgi/content/full/jem.20100559/DC1.

We thank Jeannette Scholl for excellent technical assistance, Paola Luciani for the production of the indocyanine green-containing liposomes, Alessia Galgano for help with the Cytokine Array, and Carlos Ochoa for help with the animal studies.

This work was supported by National Institutes of Health grants CA69184, Swiss National Science Foundation grant 3100A0-108207, Commission of the European Communities grant LSHC-CT-2005-518178, Oncosuisse, and Krebsliga Zurich (to M. Detmar)

The authors have no conflicting financial interests.

Submitted: 19 March 2010

Accepted: 19 August 2010

\section{REFERENCES}

Adams, R.H., and K. Alitalo. 2007. Molecular regulation of angiogenesis and lymphangiogenesis. Nat. Rev. Mol. Cell Biol. 8:464-478. doi:10 $.1038 / \mathrm{nrm} 2183$

Angeli, V., F. Ginhoux, J. Llodrà, L. Quemeneur, P.S. Frenette, M. Skobe, R. Jessberger, M. Merad, and G.J. Randolph. 2006. B cell-driven lymphangiogenesis in inflamed lymph nodes enhances dendritic cell mobilization. Immunity. 24:203-215. doi:10.1016/j.immuni.2006.01.003

Bainbridge, J., B. Sivakumar, and E. Paleolog. 2006. Angiogenesis as a therapeutic target in arthritis: lessons from oncology. Curr. Pharm. Des. 12:2631-2644. doi:10.2174/138161206777698747

Baldwin, M.E., B. Catimel, E.C. Nice, S. Roufail, N.E. Hall, K.L. Stenvers, M.J. Karkkainen, K. Alitalo, S.A. Stacker, and M.G. Achen. 2001. The specificity of receptor binding by vascular endothelial growth factor-d is different in mouse and man. J. Biol. Chem. 276:19166-19171. doi:10.1074/jbc.M100097200

Baluk, P., T. Tammela, E. Ator, N. Lyubynska, M.G. Achen, D.J. Hicklin, M. Jeltsch, T.V. Petrova, B. Pytowski, S.A. Stacker, et al. 2005. Pathogenesis of persistent lymphatic vessel hyperplasia in chronic airway inflammation. J. Clin. Invest. 115:247-257.

Bhushan, M., B. McLaughlin, J.B. Weiss, and C.E. Griffiths. 1999. Levels of endothelial cell stimulating angiogenesis factor and vascular endothelial growth factor are elevated in psoriasis. Br. J. Dermatol. 141:1054-1060. doi:10.1046/j.1365-2133.1999.03205.x

Carmeliet, P. 2003. Angiogenesis in health and disease. Nat. Med. 9:653660. doi:10.1038/nm0603-653

Cursiefen, C., J. Cao, L. Chen, Y. Liu, K. Maruyama, D. Jackson, F.E. Kruse, S.J. Wiegand, M.R. Dana, and J.W. Streilein. 2004. Inhibition of hemangiogenesis and lymphangiogenesis after normal-risk corneal transplantation by neutralizing VEGF promotes graft survival. Invest. Ophthalmol. Vis. Sci. 45:2666-2673. doi:10.1167/iovs.03-1380

Danese, S., M. Sans, C. de la Motte, C. Graziani, G. West, M.H. Phillips, R. Pola, S. Rutella, J. Willis, A. Gasbarrini, and C. Fiocchi. 2006. Angiogenesis as a novel component of inflammatory bowel disease pathogenesis. Gastroenterology. 130:2060-2073. doi:10.1053/j.gastro.2006.03.054

Detmar, M., L.F. Brown, K.P. Claffey, K.T. Yeo, O. Kocher, R.W. Jackman, B. Berse, and H.F. Dvorak. 1994. Overexpression of vascular permeability factor/vascular endothelial growth factor and its receptors in psoriasis. J. Exp. Med. 180:1141-1146. doi:10.1084/jem.180.3.1141

Detmar, M., L.F. Brown, M.P. Schön, B.M. Elicker, P. Velasco, L. Richard, D. Fukumura, W. Monsky, K.P. Claffey, and R.K. Jain. 1998. Increased microvascular density and enhanced leukocyte rolling and adhesion in the skin of VEGF transgenic mice. J. Invest. Dermatol. 111:1-6. doi:10.1046/j.1523-1747.1998.00262.x

Dixelius, J., T. Makinen, M. Wirzenius, M.J. Karkkainen, C. Wernstedt, K. Alitalo, and L. Claesson-Welsh. 2003. Ligand-induced vascular endothelial growth factor receptor-3 (VEGFR-3) heterodimerization with VEGFR-2 in primary lymphatic endothelial cells regulates tyrosine phosphorylation sites. J. Biol. Chem. 278:40973-40979. doi:10.1074/ jbc.M304499200 
Guo, R., Q. Zhou, S.T. Proulx, R. Wood, R.C. Ji, C.T. Ritchlin, B. Pytowski, Z. Zhu, Y.J. Wang, E.M. Schwarz, and L. Xing. 2009. Inhibition of lymphangiogenesis and lymphatic drainage via vascular endothelial growth factor receptor 3 blockade increases the severity of inflammation in a mouse model of chronic inflammatory arthritis. Arthritis Rheum. 60:2666-2676. doi:10.1002/art.24764

Haiko, P., T. Makinen, S. Keskitalo, J. Taipale, M.J. Karkkainen, M.E. Baldwin, S.A. Stacker, M.G. Achen, and K. Alitalo. 2008. Deletion of vascular endothelial growth factor C (VEGF-C) and VEGF-D is not equivalent to VEGF receptor 3 deletion in mouse embryos. Mol. Cell. Biol. 28:4843-4850. doi:10.1128/MCB.02214-07

Halin, C., N.E. Tobler, B. Vigl, L.F. Brown, and M. Detmar. 2007. VEGF-A produced by chronically inflamed tissue induces lymphangiogenesis in draining lymph nodes. Blood. 110:3158-3167. doi:10.1182/ blood-2007-01-066811

Halin, C., H. Fahrngruber, J.G. Meingassner, G. Bold, A. LittlewoodEvans, A. Stuetz, and M. Detmar. 2008. Inhibition of chronic and acute skin inflammation by treatment with a vascular endothelial growth factor receptor tyrosine kinase inhibitor. Am. J. Pathol. 173:265-277. doi:10.2353/ajpath.2008.071074

Hamrah, P., L. Chen, Q. Zhang, and M.R. Dana. 2003. Novel expression of vascular endothelial growth factor receptor (VEGFR)-3 and VEGF-C on corneal dendritic cells. Am. J. Pathol. 163:57-68.

Hamrah, P., L. Chen, C. Cursiefen, Q. Zhang, N.C. Joyce, and M.R. Dana. 2004. Expression of vascular endothelial growth factor receptor-3 (VEGFR-3) on monocytic bone marrow-derived cells in the conjunctiva. Exp. Eye Res. 79:553-561. doi:10.1016/j.exer.2004.06.028

Hirakawa, S., S. Kodama, R. Kunstfeld, K. Kajiya, L.F. Brown, and M. Detmar. 2005. VEGF-A induces tumor and sentinel lymph node lymphangiogenesis and promotes lymphatic metastasis. J. Exp. Med. 201:1089-1099. doi:10.1084/jem.20041896

Hoshida, T., N. Isaka, J. Hagendoorn, E. di Tomaso, Y.L. Chen, B. Pytowski, D. Fukumura, T.P. Padera, and R.K. Jain. 2006. Imaging steps of lymphatic metastasis reveals that vascular endothelial growth factor-C increases metastasis by increasing delivery of cancer cells to lymph nodes: therapeutic implications. Cancer Res. 66:8065-8075. doi:10.1158/ 0008-5472.CAN-06-1392

Hvid, H., I. Teige, P.H. Kvist, L. Svensson, and K. Kemp. 2008. TPA induction leads to a Th17-like response in transgenic K14/VEGF mice: a novel in vivo screening model of psoriasis. Int. Immunol. 20:1097-1106. doi:10.1093/intimm/dxn068

Jamieson, T., D.N. Cook, R.J. Nibbs, A. Rot, C. Nixon, P. McLean, A. Alcami, S.A. Lira, M. Wiekowski, and G.J. Graham. 2005. The chemokine receptor D6 limits the inflammatory response in vivo. Nat. Immunol. 6:403-411. doi:10.1038/ni1182

Jeltsch, M., A. Kaipainen, V. Joukov, X. Meng, M. Lakso, H. Rauvala, M. Swartz, D. Fukumura, R.K. Jain, and K. Alitalo. 1997. Hyperplasia of lymphatic vessels in VEGF-C transgenic mice. Science. 276:1423-1425. doi:10.1126/science.276.5317.1423

Joukov, V., T. Sorsa, V. Kumar, M. Jeltsch, L. Claesson-Welsh, Y. Cao, O. Saksela, N. Kalkkinen, and K. Alitalo. 1997. Proteolytic processing regulates receptor specificity and activity of VEGF-C. EMBO J. 16:3898-3911. doi:10.1093/emboj/16.13.3898

Joukov, V., V. Kumar, T. Sorsa, E. Arighi, H. Weich, O. Saksela, and K. Alitalo. 1998. A recombinant mutant vascular endothelial growth factor-C that has lost vascular endothelial growth factor receptor-2 binding, activation, and vascular permeability activities. J. Biol. Chem. 273:6599-6602. doi:10.1074/jbc.273.12.6599

Kaipainen, A., J. Korhonen, T. Mustonen, V.W. van Hinsbergh, G.H. Fang, D. Dumont, M. Breitman, and K. Alitalo. 1995. Expression of the fms-like tyrosine kinase 4 gene becomes restricted to lymphatic endothelium during development. Proc. Natl. Acad. Sci. USA.92:3566-3570. doi:10.1073/ pnas.92.8.3566

Kajiya, K., S. Hirakawa, and M. Detmar. 2006. Vascular endothelial growth factor-A mediates ultraviolet B-induced impairment of lymphatic vessel function. Am. J. Pathol. 169:1496-1503. doi:10.2353/ajpath.2006 .060197

Kanazawa, S., T. Tsunoda, E. Onuma, T. Majima, M. Kagiyama, and K. Kikuchi. 2001. VEGF, basic-FGF, and TGF-beta in Crohn's disease and ulcerative colitis: a novel mechanism of chronic intestinal inflammation. Am. J. Gastroenterol. 96:822-828.

Karkkainen, M.J., A. Saaristo, L. Jussila, K.A. Karila, E.C. Lawrence, K. Pajusola, H. Bueler, A. Eichmann, R. Kauppinen, M.I. Kettunen, et al. 2001. A model for gene therapy of human hereditary lymphedema. Proc. Natl. Acad. Sci. USA. 98:12677-12682. doi:10.1073/pnas.221449198

Karpanen, T., and K. Alitalo. 2008. Molecular biology and pathology of lymphangiogenesis. Annu. Rev. Pathol. 3:367-397. doi:10.1146/annurev .pathmechdis.3.121806.151515

Kataru, R.P., K. Jung, C. Jang, H. Yang, R.A. Schwendener, J.E. Baik, S.H. Han, K. Alitalo, and G.Y. Koh. 2009. Critical role of CD11b+ macrophages and VEGF in inflammatory lymphangiogenesis, antigen clearance, and inflammation resolution. Blood. 113:5650-5659. doi:10.1182/ blood-2008-09-176776

Kerjaschki, D., H.M. Regele, I. Moosberger, K. Nagy-Bojarski, B. Watschinger, A. Soleiman, P. Birner, S. Krieger, A. Hovorka, G. Silberhumer, et al 2004. Lymphatic neoangiogenesis in human kidney transplants is associated with immunologically active lymphocytic infiltrates. J. Am. Soc Nephrol. 15:603-612. doi:10.1097/01.ASN.0000113316.52371.2E

Kiba, A., H. Sagara, T. Hara, and M. Shibuya. 2003. VEGFR-2-specific ligand VEGF-E induces non-edematous hyper-vascularization in mice. Biochem. Biophys. Res. Commun. 301:371-377. doi:10.1016/S0006-291X (02)03033-4

Koch, A.E., L.A. Harlow, G.K. Haines, E.P. Amento, E.N. Unemori, W.L. Wong, R.M. Pope, and N. Ferrara. 1994. Vascular endothelial growth factor. A cytokine modulating endothelial function in rheumatoid arthritis. J. Immunol. 152:4149-4156.

Kriehuber, E., S. Breiteneder-Geleff, M. Groeger, A. Soleiman, S.F. Schoppmann, G. Stingl, D. Kerjaschki, and D. Maurer. 2001. Isolation and characterization of dermal lymphatic and blood endothelial cells reveal stable and functionally specialized cell lineages. J. Exp. Med. 194:797-808. doi:10.1084/jem.194.6.797

Krueger, G.G., R.G. Langley, C. Leonardi, N. Yeilding, C. Guzzo, Y. Wang, L.T. Dooley, and M. Lebwohl; CNTO 1275 Psoriasis Study Group. 2007. A human interleukin-12/23 monoclonal antibody for the treatment of psoriasis. N. Engl. J. Med. 356:580-592. doi:10.1056/ NEJMoa062382

Kunstfeld, R., S. Hirakawa, Y.K. Hong, V. Schacht, B. LangeAsschenfeldt, P. Velasco, C. Lin, E. Fiebiger, X. Wei, Y. Wu, et al 2004. Induction of cutaneous delayed-type hypersensitivity reactions in VEGF-A transgenic mice results in chronic skin inflammation associated with persistent lymphatic hyperplasia. Blood. 104:1048-1057. doi:10.1182/blood-2003-08-2964

Louten, J., K. Boniface, and R. de Waal Malefyt. 2009. Development and function of TH17 cells in health and disease. J. Allergy Clin. Immunol. 123:1004-1011. doi:10.1016/j.jaci.2009.04.003

Mäkinen, T., L. Jussila, T. Veikkola, T. Karpanen, M.I. Kettunen, K.J. Pulkkanen, R. Kauppinen, D.G. Jackson, H. Kubo, S. Nishikawa, et al. 2001a. Inhibition of lymphangiogenesis with resulting lymphedema in transgenic mice expressing soluble VEGF receptor-3. Nat. Med. 7:199205. doi:10.1038/84651

Mäkinen, T., T. Veikkola, S. Mustjoki, T. Karpanen, B. Catimel, E.C. Nice, L. Wise, A. Mercer, H. Kowalski, D. Kerjaschki, et al. 2001b. Isolated lymphatic endothelial cells transduce growth, survival and migratory signals via the VEGF-C/D receptor VEGFR-3. EMBO J. 20:4762-4773. doi:10.1093/emboj/20.17.4762

Mumprecht, V., and M. Detmar. 2009. Lymphangiogenesis and cancer metastasis. J. Cell. Mol. Med. 13:1405-1416. doi:10.1111/j.1582-4934 .2009.00834.x

Nagy, J.A., E. Vasile, D. Feng, C. Sundberg, L.F. Brown, M.J. Detmar, J.A. Lawitts, L. Benjamin, X. Tan, E.J. Manseau, et al. 2002. Vascular permeability factor/vascular endothelial growth factor induces lymphangiogenesis as well as angiogenesis. J. Exp. Med. 196:1497-1506. doi:10.1084/ jem.20021244

Nair, R.P., K.C. Duffin, C. Helms, J. Ding, P.E. Stuart, D. Goldgar, J.E. Gudjonsson, Y. Li, T. Tejasvi, B.J. Feng, et al; Collaborative Association Study of Psoriasis. 2009. Genome-wide scan reveals association of psoriasis with IL-23 and NF-kappaB pathways. Nat. Genet. 41:199-204. doi:10.1038/ng.311 
Paavonen, K., P. Puolakkainen, L. Jussila, T. Jahkola, and K. Alitalo. 2000. Vascular endothelial growth factor receptor-3 in lymphangiogenesis in wound healing. Am. J. Pathol. 156:1499-1504.

Partanen, T.A., J. Arola, A. Saaristo, L. Jussila, A. Ora, M. Miettinen, S.A. Stacker, M.G. Achen, and K. Alitalo. 2000. VEGF-C and VEGF-D expression in neuroendocrine cells and their receptor, VEGFR-3, in fenestrated blood vessels in human tissues. FASEB J. 14:2087-2096. doi:10.1096/fj.99-1049com

Polzer, K., D. Baeten, A. Soleiman, J. Distler, D.M. Gerlag, P.P. Tak, G. Schett, and J. Zwerina. 2008. Tumour necrosis factor blockade increases lymphangiogenesis in murine and human arthritic joints. Ann. Rheum. Dis. 67:1610-1616. doi:10.1136/ard.2007.083394

Pytowski, B., J. Goldman, K. Persaud, Y. Wu, L. Witte, D.J. Hicklin, M. Skobe, K.C. Boardman, and M.A. Swartz. 2005. Complete and specific inhibition of adult lymphatic regeneration by a novel VEGFR-3 neutralizing antibody. J. Natl. Cancer Inst. 97:14-21. doi:10.1093/jnci/dji003

Schmittgen, T.D., and K.J. Livak. 2008. Analyzing real-time PCR data by the comparative C(T) method. Nat. Protoc. 3:1101-1108. doi:10.1038/ nprot. 2008.73

Schonthaler, H.B., R. Huggenberger, S.K. Wculek, M. Detmar, and E.F. Wagner. 2009. Systemic anti-VEGF treatment strongly reduces skin inflammation in a mouse model of psoriasis. Proc. Natl. Acad. Sci. USA. 106:21264-21269. doi:10.1073/pnas.0907550106

Stark, H.J., D. Breitkreutz, A. Limat, P. Bowden, and N.E. Fusenig. 1987. Keratins of the human hair follicle: "hyperproliferative" keratins consistently expressed in outer root sheath cells in vivo and in vitro. Differentiation. 35:236-248.

Szuba, A., M. Skobe, M.J. Karkkainen, W.S. Shin, D.P. Beynet, N.B. Rockson, N. Dakhil, S. Spilman, M.L. Goris, H.W. Strauss, et al. 2002. Therapeutic lymphangiogenesis with human recombinant VEGF-C. FASEB J. 16:1985-1987.

Tammela, T., A. Saaristo, T. Holopainen, J. Lyytikkä, A. Kotronen, M. Pitkonen, U. Abo-Ramadan, S. Ylä-Herttuala, T.V. Petrova, and K. Alitalo. 2007. Therapeutic differentiation and maturation of lymphatic vessels after lymph node dissection and transplantation. Nat. Med. 13:1458-1466. doi:10.1038/nm1689

Tammela, T., G. Zarkada, E. Wallgard, A. Murtomäki, S. Suchting, M. Wirzenius, M. Waltari, M. Hellström, T. Schomber, R. Peltonen, et al. 2008. Blocking VEGFR-3 suppresses angiogenic sprouting and vascular network formation. Nature. 454:656-660. doi:10.1038/nature07083

Valtola, R., P. Salven, P. Heikkilä, J. Taipale, H. Joensuu, M. Rehn, T. Pihlajaniemi, H. Weich, R. deWaal, and K. Alitalo. 1999. VEGFR-3 and its ligand VEGF-C are associated with angiogenesis in breast cancer. Am. J. Pathol. 154:1381-1390.

Van den Broeck, W., A. Derore, and P. Simoens. 2006. Anatomy and nomenclature of murine lymph nodes: Descriptive study and nomenclatory standardization in BALB/cAnNCrl mice. J. Immunol. Methods. 312:12-19. doi:10.1016/j.jim.2006.01.022

Wirzenius, M., T. Tammela, M. Uutela, Y. He, T. Odorisio, G. Zambruno, J.A. Nagy, H.F. Dvorak, S. Ylä-Herttuala, M. Shibuya, and K. Alitalo. 2007. Distinct vascular endothelial growth factor signals for lymphatic vessel enlargement and sprouting. J. Exp. Med. 204:1431-1440 doi:10.1084/jem.20062642

Wuest, T.R., and D.J. Carr. 2010. VEGF-A expression by HSV-1-infected cells drives corneal lymphangiogenesis. J. Exp. Med. 207:101-115. doi:10 $.1084 / \mathrm{jem} .20091385$

Xia, Y.P., B. Li, D. Hylton, M. Detmar, G.D. Yancopoulos, and J.S. Rudge. 2003. Transgenic delivery of VEGF to mouse skin leads to an inflammatory condition resembling human psoriasis. Blood. 102:161-168. doi:10.1182/blood-2002-12-3793

Yoon, Y.S., T. Murayama, E. Gravereaux, T. Tkebuchava, M. Silver, C. Curry, A. Wecker, R. Kirchmair, C.S. Hu, M. Kearney, et al. 2003. VEGF-C gene therapy augments postnatal lymphangiogenesis and ameliorates secondary lymphedema. J. Clin. Invest. 111:717-725.

Zhang, Q., Y. Lu, S.T. Proulx, R. Guo, Z. Yao, E.M. Schwarz, B.F Boyce, and L. Xing. 2007. Increased lymphangiogenesis in joints of mice with inflammatory arthritis. Arthritis Res. Ther. 9:R118. doi: $10.1186 /$ ar2326 